\title{
Glycopeptides Related to $\beta$-Endorphin Adopt Helical Amphipathic Conformations in the Presence of Lipid Bilayers.
}

\section{Supporting Information}

Muthu Dhanasekaran, Michael M. Palian, Isabel Alves, Larisa Yeomans, Charles M. Keyari, Neil E. Jacobsen, Gordon Tollin, Victor J. Hruby and Robin Polt*

The Carl S. Marvel Laboratories, Department of Chemistry The University of Arizona, Tucson, AZ 85721

*polt@u.arizona.edu 


\section{Table of Contents}

Table S1: Chemical shift values (p.p.m) of glycopeptide 9 as measured in $\mathrm{H}_{2} \mathrm{O}: \mathrm{D}_{2} \mathrm{O}(9: 1)$ at $3.2 \mathrm{mM}$ concentration, $\mathrm{pH}=4.5$ and at $15^{\circ} \mathrm{C}$.

Table S2: Chemical shift values (p.p.m) of glycopeptide 9 as measured in a 30\% TFE- $\mathrm{d}_{3} / \mathrm{H}_{2} \mathrm{O}$ mixture at $2.16 \mathrm{mM}$ concentration, $\mathrm{pH}=4.5$ and at $20^{\circ} \mathrm{C}$.

Table S3: Chemical shift values (p.p.m) of glycopeptide 9 as measured in SDS micelle at $2.85 \mathrm{mM}$ concentration, $\mathrm{pH}=4.5$ and at $25^{\circ} \mathrm{C}$.

Table S4: Chemical shift values (p.p.m) of glycopeptide 9 as measured in DMPC:DHPC(1:2) bicelle at $3.43 \mathrm{mM}$ concentration, $\mathrm{pH}=4.5$ and $25^{\circ} \mathrm{C}$.

Table S5: Chemical shift values (p.p.m) of glycopeptide 9 as measured in DMPC:DHPC(1:2) bicelle at $3.43 \mathrm{mM}$ concentration, $\mathrm{pH}=4.5$ and at $38^{\circ} \mathrm{C}$.

Table S6:Chemical shift values (p.p.m) of glycopeptide 10 as measured in $\mathrm{H}_{2} \mathrm{O}: \mathrm{D}_{2} \mathrm{O}(9: 1)$ at $3.13 \mathrm{mM}$ concentration, $\mathrm{pH}=4.5$ and at $15^{\circ} \mathrm{C}$.

Table S7: Chemical shift values (p.p.m) of glycopeptide 10 as measured in a $30 \%$ TFE- $\mathrm{d}_{3} / \mathrm{H}_{2} \mathrm{O}$ mixture at $4.12 \mathrm{mM}$ concentration, $\mathrm{pH}=4.5$ and at $20^{\circ} \mathrm{C}$.

Tabel S8: Chemical shift values (p.p.m) of glycopeptide 10 as measured in a SDS micelle at $2.52 \mathrm{mM}$ concentration, $\mathrm{pH}=4.5$ and at $25^{\circ} \mathrm{C}$.

Table S9: Chemical shift values (p.p.m) of glycopeptide 11 as measured in $\mathrm{H} 2 \mathrm{O}: \mathrm{D} 2 \mathrm{O}$ (9:1) at $2.62 \mathrm{mM}$ concentration, $\mathrm{pH}=4.5$ and at $15^{\circ} \mathrm{C}$.

Table S10: Chemical shift values (p.p.m) of glycopeptide 11 as measured in a $30 \%$ TFE- $\mathrm{d}_{3} / \mathrm{H}_{2} \mathrm{O}$ mixture at $2.77 \mathrm{mM}$ concentration, $\mathrm{pH}=4.55$ and at $20^{\circ} \mathrm{C}$.

Table S11: Chemical shift values (p.p.m) of glycopeptide 11 as measured in SDS at $3.16 \mathrm{mM}$ concentration, $\mathrm{pH}=4.5$ and at $25^{\circ} \mathrm{C}$.

Table S12: Chemical shift values (p.p.m) of glycopeptide 12 as measured in $\mathrm{H} 2 \mathrm{O}: \mathrm{D} 2 \mathrm{O}(9: 1)$ at $2.47 \mathrm{mM}$ concentration, $\mathrm{pH}=4.5$ and at $15^{\circ} \mathrm{C}$.

Table S13: Chemical shift values (p.p.m) of glycopeptide 12 as measured in a $30 \%$ TFE- $\mathrm{d}_{3} / \mathrm{H}_{2} \mathrm{O}$ mixture at $2.75 \mathrm{mM}$ concentration, $\mathrm{pH}=4.5$ and at $20^{\circ} \mathrm{C}$.

Table S14: Chemical shift values (p.p.m) of glycopeptide 12 as measured in SDS at 2.3mM concentration, $\mathrm{pH}=4.5$ and at $25^{\circ} \mathrm{C}$.

Table S15: Chemical shift values (p.p.m) of peptide $9 \mathrm{u}$ as measured in SDS at $2.5 \mathrm{mM}$ concentration, $\mathrm{pH}=4.5$ and at $25^{\circ} \mathrm{C}$.

Figure S1: Far-UV CD spectra of a) glycopeptide 9 and b) peptide $9 u$ as a function of TFE concentration.

Figure S2: Far-UV CD spectra of a) glycopeptide 10 and b) peptide 10u as a function of TFE concentration.

Figure S3: Far-UV CD spectra of a) glycopeptide 11 and b) peptide $11 \mathrm{u}$ as a function of TFE concentration.

Figure S4: Finger print $(\alpha \mathrm{H}-\mathrm{NH})$ region of TOCSY (mixing time $=100 \mathrm{~ms})$ spectra of glycopeptide 9 in $\mathrm{H}_{2} \mathrm{O}: \mathrm{D}_{2} \mathrm{O}(9: 1)$ at $\mathrm{pH}=4.5$ at $20^{\circ} \mathrm{C}$.

Figure S5: Finger print $(\alpha \mathrm{H}-\mathrm{NH})$ region of TOCSY (mixing time $=100 \mathrm{~ms})$ spectra of glycopeptide 11 in $\mathrm{H}_{2} \mathrm{O}: \mathrm{D}_{2} \mathrm{O}(9: 1)$ at $\mathrm{pH}=4.5$ at $20^{\circ} \mathrm{C}$.

Figure S6: Finger print $(\alpha \mathrm{H}-\mathrm{NH})$ region of TOCSY (mixing time $=100 \mathrm{~ms})$ spectra of glycopeptide 12 in $\mathrm{H}_{2} \mathrm{O}: \mathrm{D}_{2} \mathrm{O}(9: 1)$ at $\mathrm{pH}=4.5$ at $20^{\circ} \mathrm{C}$.

Figure S7. Finger print $(\alpha \mathrm{H}-\mathrm{NH})$ region of TOCSY (mixing time $=100 \mathrm{~ms}$ ) spectra of glycopeptide 9 in TFE:water (3:7) at $\mathrm{pH}=4.5$ and at $15^{\circ} \mathrm{C}$.

Figure S8. Finger print $(\alpha \mathrm{H}-\mathrm{NH})$ region of TOCSY (mixing time $=100 \mathrm{~ms}$ ) spectra of glycopeptide 11 in TFE:water (3:7) at $\mathrm{pH}=4.5$ and at $15^{\circ} \mathrm{C}$.

Figure S9. Finger print $(\alpha \mathrm{H}-\mathrm{NH})$ region of TOCSY (mixing time $=100 \mathrm{~ms})$ spectra of glycopeptide 12 in TFE:water (3:7) at $\mathrm{pH}=4.5$ and at $15^{\circ} \mathrm{C}$.

Figure S10. Finger print region of $(\alpha \mathrm{H}-\mathrm{NH})$ of TOCSY (mixing time $=100 \mathrm{~ms}$ ) spectra of glycopeptide 9 in SDS micelle at $\mathrm{pH}=4.5$ and at $25^{\circ} \mathrm{C}$.

Figure S11. Finger print region of $(\alpha \mathrm{H}-\mathrm{NH})$ of TOCSY (mixing time $=100 \mathrm{~ms}$ ) spectra of glycopeptide 11 in SDS micelle at $\mathrm{pH}=4.5$ and at $25^{\circ} \mathrm{C}$. 
Figure S12. Finger print region of $(\alpha \mathrm{H}-\mathrm{NH})$ of TOCSY (mixing time $=100 \mathrm{~ms}$ ) spectra of glycopeptide 12 in SDS micelle at $\mathrm{pH}=4.5$ and at $25^{\circ} \mathrm{C}$.

Figure S13: Finger print $(\alpha \mathrm{H}-\mathrm{NH})$ region of TOCSY (mixing time $=80 \mathrm{~ms}$ ) spectra in zwitterionic bicelle at $\mathrm{pH}=4.5$ and at $25^{\circ} \mathrm{C}$.

Figure S14: Finger print $(\alpha \mathrm{H}-\mathrm{NH})$ region of NOESY (mixing time $=300 \mathrm{~ms}$ ) spectra in zwitterionic bicelle at $\mathrm{pH} 4.5$ and $38^{\circ} \mathrm{C}$.

Figure S15: Finger print $(\alpha \mathrm{H}-\mathrm{NH})$ region of TOCSY (mixing time $=80 \mathrm{~ms}$ ) spectra in zwitterionic bicelle at $\mathrm{pH} 4.5$ and $38^{\circ} \mathrm{C}$.

Figure S16. Amide $(\mathrm{NH}-\mathrm{NH})$ region of NOESY (mixing time $=300 \mathrm{~ms}$ ) spectra in zwitterionic bicelle at pH4.5 and $38^{\circ} \mathrm{C}$.

Figure S17. A portion of TOCSY (mixing time $=100 \mathrm{~ms}$ ) spectra showing all the spin system of peptide 9u in TFE:water (3:7) at $\mathrm{pH}=4.5$ and $\mathrm{at} 15^{\circ} \mathrm{C}$.

Figure S18. Finger print $(\alpha \mathrm{H}-\mathrm{NH})$ region of TOCSY (in black colour, mixing time $=100 \mathrm{~ms}$ ) and ROESY (in red color, mixing time $=150 \mathrm{~ms}$ ) of peptide $9 \mathrm{u}$ in TFE: water $(3: 7)$ at $\mathrm{pH}=4.5$ and at $15^{\circ} \mathrm{C}$.

Figure S19. Amide $(\mathrm{NH}-\mathrm{NH})$ region of ROESY (mixing time $=150 \mathrm{~ms}$ ) spectra of peptide $9 \mathrm{u}$ in TFE:water (3:7) at $\mathrm{pH}=4.5$ and at $15^{\circ} \mathrm{C}$.

Figure S20: Finger print $(\alpha \mathrm{H}-\mathrm{NH})$ region of a) TOCSY (mixing time $=100 \mathrm{~ms})$ and $b$ ) NOESY (mixing time $=300 \mathrm{~ms}$ ) of peptide $9 \mathrm{u}$ in SDS micelles at $\mathrm{pH}=4.5$ and at $25^{\circ} \mathrm{C}$.

Figure S21. Amide $(\mathrm{NH}-\mathrm{NH})$ region of NOESY (mixing time $=300 \mathrm{~ms}$ ) spectra of peptide $9 \mathrm{u}$ in SDS micelles at $\mathrm{pH}=4.5$ and at $25^{\circ} \mathrm{C}$.

Figure S22. A portion of TOCSY (mixing time $=100 \mathrm{~ms}$ ) spectra showing all the spin system of peptide 11u in TFE:water (3:7) at $\mathrm{pH}=4.5$ and at $15^{\circ} \mathrm{C}$.

Figure S23. Finger print $(\alpha \mathrm{H}-\mathrm{NH})$ region of TOCSY (in black color, mixing time $=100 \mathrm{~ms}$ ) and ROESY (in red colour, mixing time $=150 \mathrm{~ms}$ ) of peptide $11 \mathrm{u}$ in TFE:water (3:7) at $\mathrm{pH}=4.5$ and at $15^{\circ} \mathrm{C}$.

Figure S24. Amide (NH-NH) region of ROESY (mixing time $=150 \mathrm{~ms}$ ) spectra of peptide $11 \mathrm{u}$ in TFE:water (3:7) at $\mathrm{pH}=4.5$ and at $15^{\circ} \mathrm{C}$.

Figure S25. Summary of NOEs observed for peptide $9 \mathrm{u}$ in SDS micelle at $\mathrm{pH}=4.5$ and $25^{\circ} \mathrm{C}$.

Figure S26. A portion of ${ }^{1} \mathrm{H}$ NMR spectra displaying amide and aromatic proton assignments in $\mathrm{H}_{2} \mathrm{O} / \mathrm{D}_{2} \mathrm{O}$ $(9: 1)$ at $\mathrm{pH}=4.5$ and $20^{\circ} \mathrm{C}$.

Figure S27. Amide $(\mathrm{NH}-\mathrm{NH})$ region of ROESY (mixing time $=150 \mathrm{~ms}$ ) spectra displaying $\mathrm{d}_{\mathrm{NN}}(\mathrm{i}, \mathrm{i}+1)$ ROE peaks in $\mathrm{H}_{2} \mathrm{O}: \mathrm{D}_{2} \mathrm{O}(9: 1)$ at $\mathrm{pH}=4.5$ at $20^{\circ} \mathrm{C}$.

Figure S28. A portion of ${ }^{1} \mathrm{H}$ NMR spectra displaying amide and aromatic proton assignments in $\mathrm{TFE} / \mathrm{H}_{2} \mathrm{O}$ (3:7) and $\mathrm{pH}=4.5$ at $15^{\circ} \mathrm{C}$.

Figure S29. Amide $(\mathrm{NH}-\mathrm{NH})$ region of ROESY (mixing time $=150 \mathrm{msec}$ ) displaying $\mathrm{d}_{\mathrm{NN}}(\mathrm{i}, \mathrm{i}+1)$ NOEs spectra in TFE $/ \mathrm{H}_{2} \mathrm{O}(3: 7)$ and $\mathrm{pH}=4.5$ at $15^{\circ} \mathrm{C}$.

Figure S30. Fingerprint region $(\alpha \mathrm{H}-\mathrm{NH})$ of ROESY (mixing time $=150 \mathrm{~ms})$ spectra in TFE: water (3:7) and $\mathrm{pH}=4.5$ at $15^{\circ} \mathrm{C}$.

Figure S31. A portion of ${ }^{1} \mathrm{H}$ NMR spectra displaying amide and aromatic proton assignments in SDS micelles at $\mathrm{pH}=4.5$ at $25^{\circ} \mathrm{C}$.

Figure S32. Amide region $(\mathrm{NH}-\mathrm{NH})$ of NOESY (mixing time $=300 \mathrm{msec}$ ) spectra in SDS micelles at $\mathrm{pH}=4.5$ and $25^{\circ} \mathrm{C}$.

Figure S33. Finger print region $(\alpha \mathrm{H}-\mathrm{NH})$ of NOESY spectra in SDS micelles at $\mathrm{pH}=4.5$ and $25^{\circ} \mathrm{C}$.

Figure S34. Lowest energy structures for 9-12. 
Table S1: Chemical shift values (p.p.m) of glycopeptide 9 as measured in $\mathrm{H}_{2} \mathrm{O}: \mathrm{D}_{2} \mathrm{O}$ (9:1) at $3.2 \mathrm{mM}$ concentration, $\mathrm{pH}=4.5$ and at $15^{\circ} \mathrm{C}$.

\begin{tabular}{|c|c|c|c|c|c|}
\hline Residue & $\mathrm{NH}$ & $\mathrm{C}^{\alpha} \mathrm{H}$ & $\mathrm{C}^{\beta} \mathrm{H}$ & Others & $\begin{array}{l}\Delta \delta \text { (Obs.-r.c.) } \\
\text { ppm }\end{array}$ \\
\hline Tyr1 & & 4.334 & $\begin{array}{l}3.135, \\
3.193\end{array}$ & $\delta \mathrm{CH}: 7.258,7.245$ & -0.226 \\
\hline DThr2 & 8.581 & 4.166 & 4.105 & $\gamma \mathrm{CH}_{2}: 0.893$ & -0.154 \\
\hline Gly3 & 8.468 & 3.885 & & & -0.125 \\
\hline Phe4 & 8.126 & 4.628 & $\begin{array}{l}3.020, \\
3.078\end{array}$ & 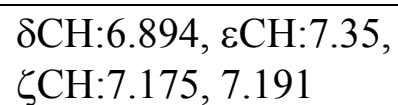 & 0.008 \\
\hline Leu5 & 8.296 & 4.612 & 1.586 & $\begin{array}{l}\gamma \mathrm{CH}_{2}: 1.544 \\
\delta \mathrm{CH}_{3}: 0.921\end{array}$ & 0.262 \\
\hline Pro6 & - & 4.332 & $2.01,2.29$ & $\begin{array}{l}\gamma \mathrm{CH}_{2}: 1.882 \\
\delta \mathrm{CH}_{2}: 3.687,3.630\end{array}$ & -0.108 \\
\hline Asn7 & 8.541 & 4.649 & $\begin{array}{l}2.797, \\
2.853\end{array}$ & & -0.111 \\
\hline Leu8 & 8.255 & 4.217 & 1.671 & $\begin{array}{l}\gamma \mathrm{CH}_{2}: 1.604 \\
\delta \mathrm{CH}_{3}: 0.870,0.945\end{array}$ & -0.133 \\
\hline Aib9 & 8.274 & - & 1.463 & & \\
\hline Glu10 & 8.255 & 4.176 & $\begin{array}{l}2.034 \\
2.096\end{array}$ & $\gamma \mathrm{CH}_{2}: 2.346$ & -0.114 \\
\hline Lys11 & 8.086 & 4.238 & 1.914 & $\begin{array}{l}\gamma \mathrm{CH}_{2}: 1.697 \\
\delta \mathrm{CH}_{2}: 1.836\end{array}$ & -0.082 \\
\hline Ala12 & 7.981 & 4.288 & 1.414 & & -0.052 \\
\hline Leu13 & 8.101 & 4.318 & 1.682 & $\begin{array}{l}\gamma \mathrm{CH}_{2}: 1.589 \\
\delta \mathrm{CH}_{3}: 0.894,0.948\end{array}$ & -0.032 \\
\hline Lys 14 & 8.288 & 4.329 & 1.854 & $\begin{array}{l}\gamma \mathrm{CH}_{2}: 1.699 \\
\delta \mathrm{CH}_{2}: 1.794\end{array}$ & 0.009 \\
\hline Ser15 & 8.566 & 4.630 & $\begin{array}{l}3.954, \\
4.253\end{array}$ & & 0.14 \\
\hline Leu16 & 8.360 & 4.321 & 1.708 & $\begin{array}{l}\gamma \mathrm{CH}_{2}: 1.625 \\
\delta \mathrm{CH}_{3}: 0.885,0.949\end{array}$ & -0.029 \\
\hline$-\mathrm{CONH}_{2}$ & $\begin{array}{l}7.249, \\
7.447\end{array}$ & & & & \\
\hline
\end{tabular}


Table S2: Chemical shift values (p.p.m) of glycopeptide 9 as measured in a 30\% TFE$\mathrm{d}_{3} / \mathrm{H}_{2} \mathrm{O}$ mixture at $2.16 \mathrm{mM}$ concentration, $\mathrm{pH}=4.5$ and at $20^{\circ} \mathrm{C}$.

\begin{tabular}{|c|c|c|c|c|c|}
\hline Residue & $\mathrm{NH}$ & $\mathrm{C}^{\alpha} \mathrm{H}$ & $\mathrm{C}^{\beta} \mathrm{H}$ & Others & $\begin{array}{l}\Delta \delta(\text { Obs.- } \\
\text { r.c.) ppm }\end{array}$ \\
\hline Tyr1 & & 4.302 & $3.177,3.127$ & $\begin{array}{l}\delta \mathrm{CH}: 7.188, \\
7.200, \\
\varepsilon \mathrm{CH}: 6.898\end{array}$ & -0.258 \\
\hline DThr2 & n.o. & 4.193 & 4.168 & & -0.197 \\
\hline Gly3 & 8.347 & $3.910,3.960$ & & & -0.075 \\
\hline Phe4 & 8.012 & 4.669 & 3.102 & $\begin{array}{l}\text { ¿CH:7.268 } \\
\varepsilon \mathrm{CH}: 7.040\end{array}$ & 0.049 \\
\hline Leu5 & 7.917 & 4.559 & 1.632 & $\begin{array}{l}\gamma \mathrm{CH}_{2}: 1.632 \\
\delta \mathrm{CH}_{3}: 0.943, \\
0.969\end{array}$ & 0.209 \\
\hline Pro6 & & 4.299 & 2.320 & $\begin{array}{l}\gamma \mathrm{CH}_{2}: 2.055 \\
\delta \mathrm{CH}_{2}: 3.535, \\
3.666\end{array}$ & -0.141 \\
\hline Asn7 & 8.169 & 4.595 & 2.847 & $\begin{array}{l}\delta \mathrm{NH}: 7.617, \\
6.824\end{array}$ & -0.165 \\
\hline Leu8 & 7.845 & 4.153 & 1.674 & $\begin{array}{l}\gamma \mathrm{CH}_{2}: 1.674 \\
\delta \mathrm{CH}_{3}: 0.903, \\
0.960\end{array}$ & -0.197 \\
\hline Aib9 & 8.038 & & 1.493 & & \\
\hline Glu10 & 8.068 & 3.930 & $2.306,2.384$ & $\gamma \mathrm{CH}_{2}: 2.11$ & -0.36 \\
\hline Lys11 & 7.792 & 4.075 & 1.997 & $\begin{array}{l}\gamma \mathrm{CH}_{2}: 1.520 \\
\delta \mathrm{CH}_{2}: 1.640, \\
1.722\end{array}$ & -0.245 \\
\hline Ala12 & 8.006 & 4.243 & 1.494 & & -0.097 \\
\hline Leu13 & 8.130 & 4.218 & 1.838 & $\begin{array}{l}\gamma \mathrm{CH}_{2}: 1.574 \\
\delta \mathrm{CH}_{3} \text { :n.o. }\end{array}$ & -0.132 \\
\hline Lys 14 & 7.922 & 4.203 & 1.966 & $\begin{array}{l}\gamma \mathrm{CH}_{2}: 1.491 \\
\delta \mathrm{CH}_{2}: 1.741\end{array}$ & -0.117 \\
\hline Ser15 & 7.991 & 4.611 & 4.045 & & 0.121 \\
\hline Leu16 & 7.982 & 4.310 & 1.816 & $\begin{array}{l}\gamma \mathrm{CH}_{2}: 1.650 \\
\delta \mathrm{CH}_{3}: 0.909, \\
0.961\end{array}$ & -0.04 \\
\hline$-\mathrm{CONH}_{2}$ & $7.045,7.268$ & & & & \\
\hline
\end{tabular}


Table S3: Chemical shift values (p.p.m) of glycopeptide 9 as measured in SDS micelle at $2.85 \mathrm{mM}$ concentration, $\mathrm{pH}=4.5$ and at $25^{\circ} \mathrm{C}$.

\begin{tabular}{|c|c|c|c|c|c|}
\hline Residue & $\mathrm{NH}$ & $\mathrm{C}^{\alpha} \mathrm{H}$ & $\mathrm{C}^{\beta} \mathrm{H}$ & Others & $\begin{array}{l}\Delta \delta \text { (Obs-R.C.) } \\
\text { ppm }\end{array}$ \\
\hline Tyr1 & & 4.446 & $3.051,3.226$ & $\delta \mathrm{CH}: 7.21, \varepsilon \mathrm{CH}: 6.85$ & -0.114 \\
\hline DThr2 & 8.608 & 4.334 & 4.334 & $\gamma \mathrm{CH}_{2}: 1.147$ & -0.056 \\
\hline Gly3 & 8.431 & 3.947 & & & -0.063 \\
\hline Phe4 & 7.908 & 4.545 & $3.056,3.231$ & $\begin{array}{l}\delta \mathrm{CH}: 6.92, \varepsilon \mathrm{CH}: 7.257, \\
\zeta \mathrm{CH}: 7.211\end{array}$ & -0.075 \\
\hline Leu5 & 8.163 & 4.254 & 1.876 & $\begin{array}{l}\gamma \mathrm{CH}_{2}: 1.548,1.673 \\
\delta \mathrm{CH}_{3}: 0.984\end{array}$ & -0.096 \\
\hline Pro6 & & 4.20 & 2.11 & $\begin{array}{l}\gamma \mathrm{CH}_{2}: 1.86 \\
\delta \mathrm{CH}_{2}: 3.58 \\
\end{array}$ & -0.24 \\
\hline Asn7 & 7.563 & 4.451 & $2.854,2.757$ & $\delta \mathrm{NH}: 6.85,7.26$ & -0.309 \\
\hline Leu8 & 7.853 & 3.964 & s.o. & $\begin{array}{l}\gamma \mathrm{CH}_{2}: 1.560 \\
\delta \mathrm{CH}_{3}: 0.985 \\
\end{array}$ & -0.386 \\
\hline Aib9 & 8.423 & - & 1.474 & & \\
\hline Glu10 & 7.802 & 3.957 & 2.164 & $\gamma \mathrm{CH}_{2}: 2.511,2.586$ & -0.333 \\
\hline Lys11 & 7.836 & 4.007 & 2.001 & $\begin{array}{l}\gamma \mathrm{CH}_{2} \text { :s.o. } \\
\delta \mathrm{CH}_{2} \text { :s.o. }\end{array}$ & -0.313 \\
\hline Ala12 & 7.974 & 4.176 & 1.448 & & -0.164 \\
\hline Leu13 & 8.028 & 4.123 & 1.790 & $\begin{array}{l}\gamma \mathrm{CH}_{2}: 1.613 \\
\delta \mathrm{CH}_{3}: 0.891 \\
\end{array}$ & -0.227 \\
\hline Lys 14 & 7.861 & 4.113 & 1.904 & $\begin{array}{l}\gamma \mathrm{CH}_{2}: 1.434 \\
\delta \mathrm{CH}_{2}: 1.695\end{array}$ & -0.207 \\
\hline Ser15 & 7.933 & 4.606 & $4.078,4.168$ & & 0.116 \\
\hline Leu16 & 7.911 & 4.230 & 1.774 & $\begin{array}{l}\gamma \mathrm{CH}_{2}: 1.593 \\
\delta \mathrm{CH}_{3}: 0.884,0.909\end{array}$ & -0.12 \\
\hline$-\mathrm{CONH}_{2}$ & 6.926, & & & & \\
\hline
\end{tabular}

s.o.= signal overlab 
Table S4: Chemical shift values (p.p.m) of glycopeptide 9 as measured in DMPC:DHPC(1:2) bicelle at $3.43 \mathrm{mM}$ concentration, $\mathrm{pH}=4.5$ and at $25^{\circ} \mathrm{C}$.

\begin{tabular}{|c|c|c|c|c|c|}
\hline Residue & $\mathrm{NH}$ & $\mathrm{C}^{\alpha} \mathrm{H}$ & $\mathrm{C}^{\beta} \mathrm{H}$ & Others & $\begin{array}{l}\Delta \delta \text { (Obs-R.C.) } \\
\text { ppm }\end{array}$ \\
\hline Tyr1 & & 4.341 & $3.112,3.168$ & $\delta \mathrm{CH}: 7.168, \varepsilon \mathrm{CH}: 6.85$ & -0.219 \\
\hline DThr2 & 8.566 & 4.176 & 4.139 & $\gamma \mathrm{CH}_{2}: 0.925$ & -0.214 \\
\hline Gly3 & 8.463 & $\begin{array}{l}3.925, \\
3.973\end{array}$ & & & -0.037 \\
\hline Phe4 & 8.250 & 4.555 & $3.071,3.184$ & 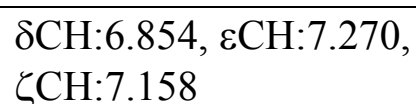 & -0.065 \\
\hline Leu5 & 8.505 & 4.340 & $1.777,1.653$ & $\begin{array}{l}\gamma \mathrm{CH}_{2}: 1.537 \\
\delta \mathrm{CH}_{3}: 0.906,0.955\end{array}$ & -0.01 \\
\hline Pro6 & - & 4.241 & s.o. & $\begin{array}{l}\gamma \mathrm{CH}_{2} \text { :s.o. } \\
\delta \mathrm{CH}_{2} \text { :s.o. }\end{array}$ & -0.199 \\
\hline Asn7 & 8.011 & 4.506 & $2.778,2.838$ & $\delta \mathrm{NH}: 6.919,7.651$ & -0.254 \\
\hline Leu8 & 8.004 & 4.046 & 1.909 & $\begin{array}{l}\gamma \mathrm{CH}_{2}: 1.611 \\
\delta \mathrm{CH}_{3}: 0.893\end{array}$ & -0.304 \\
\hline Aib9 & 8.305 & - & 1.408 & & \\
\hline Glu10 & 8.036 & 3.961 & 2.12 & $\gamma \mathrm{CH}_{2}: 2.356,2.438$ & -0.329 \\
\hline Lys11 & 7.954 & 4.052 & 1.913 & $\begin{array}{l}\gamma \mathrm{CH}_{2}: 1.482 \\
\delta \mathrm{CH}_{2}: 1.612,1.690\end{array}$ & -0.268 \\
\hline Ala12 & 8.088 & 4.208 & 1.430 & & -0.132 \\
\hline Leu13 & 8.008 & 4.252 & 1.909 & $\begin{array}{l}\gamma \mathrm{CH}_{2}: 1.611 \\
\delta \mathrm{CH}_{3}: 0.909\end{array}$ & -0.098 \\
\hline Lys 14 & 7.995 & 4.142 & 1.909 & $\begin{array}{l}\gamma \mathrm{CH}_{2}: 1.476 \\
\delta \mathrm{CH}_{2}: 1.611\end{array}$ & -0.178 \\
\hline Ser15 & 8.011 & 4.603 & 4.200 & & 0.113 \\
\hline Leu16 & 7.910 & 4.221 & 1.809 & $\begin{array}{l}\gamma \mathrm{CH}_{2}: 1.603 \\
\delta \mathrm{CH}_{3}: 0.875,0.910\end{array}$ & -0.129 \\
\hline$-\mathrm{CONH}_{2}$ & $\begin{array}{l}7.059, \\
7.199\end{array}$ & & & & \\
\hline
\end{tabular}

s.o.= signal overlab 
Table S5: Chemical shift values (p.p.m) of glycopeptide 9 as measured in DMPC:DHPC(1:2) bicelle at $3.43 \mathrm{mM}$ concentration, $\mathrm{pH}=4.5$ and at $38^{\circ} \mathrm{C}$.

\begin{tabular}{|c|c|c|c|c|c|}
\hline Residue & $\mathrm{NH}$ & $\mathrm{C}^{\alpha} \mathrm{H}$ & $\mathrm{C}^{\beta} \mathrm{H}$ & Others & $\begin{array}{l}\Delta \delta \text { (Obs-R.C.) } \\
\text { ppm }\end{array}$ \\
\hline Tyr1 & & 4.343 & $3.123,3.185$ & $\delta \mathrm{CH}: 7.167, \varepsilon \mathrm{CH}: 6.852$ & -0.217 \\
\hline DThr2 & 8.391 & 4.189 & 4.148 & $\gamma \mathrm{CH}_{2}$ :s.o. & -0.242 \\
\hline Gly3 & 8.391 & $\begin{array}{l}3.929, \\
3.987\end{array}$ & & & -0.023 \\
\hline Phe4 & 8.184 & 4.571 & $3.071,3.180$ & $\begin{array}{l}\text { 8CH:6.857, \&CH:7.270, } \\
\zeta \mathrm{CH}: 7.157\end{array}$ & -0.049 \\
\hline Leu5 & 8.385 & 4.338 & 1.759 & $\begin{array}{l}\gamma \mathrm{CH}_{2}: 1.641 \\
\delta \mathrm{CH}_{3}: 0.901,0.943\end{array}$ & -0.012 \\
\hline Pro6 & - & 4.238 & s.o. & $\begin{array}{l}\gamma \mathrm{CH}_{2} \text { :s.o. } \\
\delta \mathrm{CH}_{2} \text { :s.o. }\end{array}$ & -0.202 \\
\hline Asn7 & 7.918 & 4.509 & $2.784,2.831$ & $\delta \mathrm{NH}: 6.858,7.573$ & -0.251 \\
\hline Leu8 & 7.955 & 4.048 & 1.803 & $\begin{array}{l}\gamma \mathrm{CH}_{2}: 1.624 \\
\delta \mathrm{CH}_{3}: 0.871,0.912\end{array}$ & -0.302 \\
\hline Aib9 & 8.228 & - & 1.404 & & \\
\hline Glu10 & 7.970 & 3.962 & 2.124 & $\gamma \mathrm{CH}_{2}: 2.437,2.357$ & -0.328 \\
\hline Lys11 & 7.893 & 4.057 & 1.912 & $\begin{array}{l}\gamma \mathrm{CH}_{2}: 1.482 \\
\delta \mathrm{CH}_{2}: 1.609 \\
\end{array}$ & -0.263 \\
\hline Ala12 & 8.027 & 4.215 & 1.426 & & -0.125 \\
\hline Leu13 & 7.934 & 4.265 & 1.803 & $\begin{array}{l}\gamma \mathrm{CH}_{2}: 1.624 \\
\delta \mathrm{CH}_{3}: 0.893\end{array}$ & -0.085 \\
\hline Lys14 & 7.919 & 4.149 & 1.915 & $\begin{array}{l}\gamma \mathrm{CH}_{2}: 1.475 \\
\delta \mathrm{CH}_{2}: 1.609 \\
\end{array}$ & -0.171 \\
\hline Ser15 & 7.963 & 4.619 & 4.201 & & 0.129 \\
\hline Leu16 & 7.853 & 4.233 & 1.794 & $\begin{array}{l}\gamma \mathrm{CH}_{2}: 1.609 \\
\delta \mathrm{CH}_{3}: 0.871,0.908\end{array}$ & -0.117 \\
\hline$-\mathrm{CONH}_{2}$ & $\begin{array}{l}6.965, \\
7.168\end{array}$ & & & & \\
\hline
\end{tabular}

s.o.= signal overlab 
Table S6:Chemical shift values (p.p.m) of glycopeptide $\mathbf{1 0}$ as measured in $\mathrm{H}_{2} \mathrm{O}: \mathrm{D}_{2} \mathrm{O}$ (9:1) at $3.13 \mathrm{mM}$ concentration, $\mathrm{pH}=4.5$ and at $15^{\circ} \mathrm{C}$.

\begin{tabular}{|c|c|c|c|c|c|}
\hline Residue & $\mathrm{NH}$ & $\mathrm{C}^{\alpha} \mathrm{H}$ & $\mathrm{C}^{\beta} \mathrm{H}$ & Others & $\begin{array}{l}\Delta \delta(\text { Obs.- } \\
\text { r.c.) ppm }\end{array}$ \\
\hline Tyr1 & - & 4.336 & $3.121,3.187$ & $\begin{array}{l}\text { 8CH: 7.188, } \\
6.90\end{array}$ & -0.224 \\
\hline DThr2 & 8.603 & 4.170 & 4.114 & $\gamma \mathrm{CH}_{3}: 0.897$ & -0.22 \\
\hline Gly3 & 8.494 & 3.889 & & & -0.121 \\
\hline Phe4 & 8.156 & 4.593 & 3.063 & $\begin{array}{l}\mathrm{CH}: 6.874, \\
\gamma \mathrm{CH}: 7.258, \\
\varepsilon \mathrm{CH}: 7.164\end{array}$ & -0.027 \\
\hline Leu5 & 8.267 & 4.224 & 1.522 & $\begin{array}{l}\gamma \mathrm{CH}_{2}: 1.522 \\
\delta \mathrm{CH}_{3}: 0.847, \\
0.902\end{array}$ & -0.126 \\
\hline$\beta$ Ala6 & 7.826 & 2.468 & $3.358,3.424$ & : & \\
\hline Asn 7 & 8.439 & 4.659 & $2.745,2.813$ & $\begin{array}{l}\text { 8NH:6.973, } \\
7.659\end{array}$ & -0.101 \\
\hline Leu8 & 8.401 & 4.199 & 1.657 & $\begin{array}{l}\gamma \mathrm{CH}_{2}: 1.587 \\
\delta \mathrm{CH}_{3}: 0.877, \\
0.939\end{array}$ & -0.151 \\
\hline Aib9 & 8.336 & & 1.454 & & \\
\hline Glu10 & 8.208 & 4.171 & 2.357 & $\begin{array}{l}\gamma \mathrm{CH}_{2}: 2.031, \\
2.098\end{array}$ & -0.119 \\
\hline Lys11 & 8.088 & 4.228 & 1.687 & $\begin{array}{l}\gamma \mathrm{CH}_{2}: 1.829 \\
\delta \mathrm{CH}_{2}: 1.909\end{array}$ & -0.092 \\
\hline Ala12 & 7.992 & 4.287 & 1.416 & & -0.053 \\
\hline Leu13 & 8.097 & 4.319 & 1.687 & $\begin{array}{l}\gamma \mathrm{CH}_{2}: 1.590 \\
\delta \mathrm{CH}_{3}: 0.896, \\
0.953\end{array}$ & -0.031 \\
\hline Lys 14 & 8.289 & 4.328 & 1.851 & $\begin{array}{l}\gamma \mathrm{CH}_{2}: 1.464 \\
\delta \mathrm{CH}_{2}: 1.694, \\
1.851\end{array}$ & 0.008 \\
\hline Ser15 & 8.563 & 4.624 & $3.948,4.246$ & & 0.134 \\
\hline Leu16 & 8.358 & 4.317 & 1.705 & $\begin{array}{l}\gamma \mathrm{CH}_{2}: 1.629 \\
\delta \mathrm{CH}_{3}: 0.888, \\
0.949\end{array}$ & -0.033 \\
\hline$-\mathrm{CONH}_{2}$ & 7.444 & & & & \\
\hline
\end{tabular}


Table S7: Chemical shift values (p.p.m) of glycopeptide $\mathbf{1 0}$ as measured in a 30\% TFE$\mathrm{d}_{3} / \mathrm{H}_{2} \mathrm{O}$ mixture at $4.12 \mathrm{mM}$ concentration, $\mathrm{pH}=4.5$ and at $20^{\circ} \mathrm{C}$.

\begin{tabular}{|c|c|c|c|c|c|}
\hline Residue & $\mathrm{NH}$ & $\mathrm{C}^{\alpha} \mathrm{H}$ & $\mathrm{C}^{\beta} \mathrm{H}$ & Others & $\begin{array}{l}\Delta \delta(\text { Obs.- } \\
\text { r.c.) ppm }\end{array}$ \\
\hline Tyr1 & - & 4.338 & $3.176,3.201$ & $\begin{array}{l}\text { 8CH:7.217, } \\
\varepsilon \mathrm{CH}: 6.927\end{array}$ & -0.222 \\
\hline DThr2 & 8.508 & 4.290 & 4.226 & $\gamma \mathrm{CH}_{3}: 0.0 .934$ & -0.10 \\
\hline Gly3 & 8.513 & $3.929,4.011$ & & & -0.04 \\
\hline Phe4 & 8.142 & 4.558 & $3.068,3.181$ & $\begin{array}{l}7.015,7.280, \\
7.437\end{array}$ & -0.062 \\
\hline Leu5 & 7.901 & 4.178 & 1.658 & $\begin{array}{l}\gamma \mathrm{CH}_{2}: 1.580 \\
\delta \mathrm{CH}_{3}: 0.907, \\
0.961\end{array}$ & -0.172 \\
\hline$\beta$ Ala6 & 7.359 & 2.450 & $3.198,3.803$ & & \\
\hline Asn7 & 8.015 & 4.475 & 2.824 & $\begin{array}{l}8 \mathrm{NH}: 6.830, \\
7.450\end{array}$ & -0.285 \\
\hline Leu8 & 8.113 & 4.104 & 1.761 & $\begin{array}{l}\gamma \mathrm{CH}_{2}: 1.716 \\
\delta \mathrm{CH}_{3}: 0.899, \\
0.938\end{array}$ & -0.246 \\
\hline Aib9 & 8.229 & & 1.463 & & \\
\hline Glu10 & 8.061 & 3.944 & 2.144 & $\begin{array}{l}\gamma \mathrm{CH}_{2}: 2.526, \\
2.393\end{array}$ & -0.346 \\
\hline Lys11 & 7.956 & 4.073 & 2.034 & $\begin{array}{l}\gamma \mathrm{CH}_{2}: 1.525 \\
\delta \mathrm{CH}_{2}: 1.655 \text {, } \\
1.729\end{array}$ & -0.247 \\
\hline Ala12 & 8.103 & 4.245 & 1.528 & & -0.095 \\
\hline Leu13 & 8.196 & 4.221 & 1.862 & $\begin{array}{l}\gamma \mathrm{CH}_{2}: 1.587 \\
\delta \mathrm{CH}_{3}: 0.903\end{array}$ & -0.129 \\
\hline Lys 14 & 7.943 & 4.201 & 1.991 & $\begin{array}{l}\gamma \mathrm{CH}_{2}: 1.548 \\
\delta \mathrm{CH}_{2}: 1.632, \\
1.755\end{array}$ & -0.119 \\
\hline Ser15 & 7.992 & 4.624 & 4.063 & & 0.134 \\
\hline Leu16 & 7.988 & 4.321 & 1.846 & $\begin{array}{l}\gamma \mathrm{CH}_{2}: 1.664 \\
\delta \mathrm{CH}_{3}: 0.920, \\
0.971\end{array}$ & -0.029 \\
\hline
\end{tabular}


Tabel S8: Chemical shift values (p.p.m) of glycopeptide 10 as measured in a SDS micelle at $2.52 \mathrm{mM}$ concentration, $\mathrm{pH}=4.5$ and at $25^{\circ} \mathrm{C}$.

\begin{tabular}{|l|l|l|l|l|l|}
\hline Residue & $\mathrm{NH}$ & $\mathrm{C}^{\alpha} \mathrm{H}$ & $\mathrm{C}^{\beta} \mathrm{H}$ & Others & $\begin{array}{l}\Delta \delta \text { (Obs.-r.c.) } \\
\text { ppm }\end{array}$ \\
\hline Tyr1 & - & 4.479 & $2.971,3.313$ & $\delta \mathrm{CH}: 7.276, \varepsilon \mathrm{CH}: 6.873$ & -0.081 \\
\hline DThr2 & 8.820 & 4.502 & 1.253 & & 0.112 \\
\hline Gly3 & 8.769 & 3.981 & & & -0.029 \\
\hline Phe4 & 7.983 & 4.410 & $2.882,3.340$ & $\begin{array}{l}\delta \mathrm{CH}: 6.873, \varepsilon \mathrm{CH}: 7.260, \\
\zeta \mathrm{CH}: 7.219\end{array}$ & -0.21 \\
\hline Leu5 & 7.815 & 3.976 & 1.712 & $\begin{array}{l}\gamma \mathrm{CH}_{2}: 1.470 \\
\delta \mathrm{CH}_{3}: 0.896,0.995\end{array}$ & -0.374 \\
\hline$\beta$ Ala6 & & & & & -0.566 \\
\hline Asn7 & 7.832 & 4.194 & $2.744,2.895$ & & -0.477 \\
\hline Leu8 & 7.977 & 3.873 & 1.643 & $\begin{array}{l}\gamma \mathrm{CH}_{2}: 1.522 \\
\delta \mathrm{CH}_{3}: 0.797\end{array}$ & -0.39 \\
\hline Aib9 & 8.605 & - & $1.402,1.554$ & & -0.236 \\
\hline Glu10 & 8.041 & 3.900 & $2.077,2.141$ & $\gamma \mathrm{CH}_{2}: 2.481,2.606$ & -0.36 \\
\hline Lys11 & 7.852 & 4.084 & 1.891 & $\begin{array}{l}\gamma \mathrm{CH}_{2}: 1.443,1.537 \\
\delta \mathrm{CH}_{2}: 1.675\end{array}$ & -0.259 \\
\hline Ala12 & 8.042 & 3.980 & 1.439 & & -0.17 \\
\hline Leu13 & 8.161 & 4.091 & 1.673 & $\begin{array}{l}\gamma \mathrm{CH}_{2}: 1.673 \\
\delta \mathrm{CH}_{3}: 0.881\end{array}$ & 0.089 \\
\hline Lys14 & 8.034 & 4.150 & s.o. & $\mathrm{s.o}$ & -0.142 \\
\hline Ser15 & 7.908 & 4.579 & $4.070,4.159$ & & $\begin{array}{l}\gamma \mathrm{CH}_{2}: 1.576 \\
\delta \mathrm{CH}_{3}: 0.873\end{array}$ \\
\hline Leu16 & 7.930 & 4.208 & 1.754 & & - \\
\hline- & $6.93,7.38$ & & & & \\
\hline CONH & & & & & \\
\hline
\end{tabular}


Table S9: Chemical shift values (p.p.m) of glycopeptide 11 as measured in H2O:D2O (9:1) at $2.62 \mathrm{mM}$ concentration, $\mathrm{pH}=4.5$ and at $15^{\circ} \mathrm{C}$.

\begin{tabular}{|c|c|c|c|c|c|c|}
\hline Residue & $\mathrm{NH}$ & $\mathrm{C}^{\alpha} \mathrm{H}$ & $\mathrm{C}^{\beta} \mathrm{H}$ & Others & ${ }^{3} \mathrm{~J}_{\alpha \mathrm{NH}} \mathrm{Hz}$ & $\begin{array}{l}\Delta \delta \\
\mathrm{ppm}\end{array}$ \\
\hline Tyr1 & - & 4.333 & $\begin{array}{l}3.126, \\
3.184\end{array}$ & $\begin{array}{l}\delta \mathrm{CH}: 7.177, \\
\varepsilon \mathrm{CH}: 6.90\end{array}$ & & -0.227 \\
\hline DThr2 & 8.598 & 4.169 & 4.114 & $\gamma \mathrm{CH}_{3}: 0.892$ & & -0.221 \\
\hline Gly3 & 8.490 & 3.876 & & & & -0.134 \\
\hline Phe4 & 8.199 & 4.627 & 3.071 & $\begin{array}{l}7.27,7.21, \\
6.90\end{array}$ & & 0.007 \\
\hline Leu5 & 8.456 & 4.322 & 1.609 & $\begin{array}{l}\gamma \mathrm{CH}_{2}: 1.561 \\
\delta \mathrm{CH}_{3}: 0.849, \\
0.908\end{array}$ & & -0.028 \\
\hline Gly6 & 7.915 & 3.902 & & & & -0.108 \\
\hline Gly7 & 8.299 & 3.965 & & & & -0.045 \\
\hline Asn8 & 8.438 & 4.698 & $\begin{array}{l}2.765, \\
2.833\end{array}$ & $\begin{array}{l}\text { 8NH: } 7.66, \\
6.967\end{array}$ & & -0.062 \\
\hline Leu9 & 8.325 & 4.185 & 1.657 & $\begin{array}{l}\gamma \mathrm{CH}_{2}: 1.572 \\
\delta \mathrm{CH}_{3}: 0.866, \\
0.933\end{array}$ & & -0.165 \\
\hline Aib10 & 8.307 & & 1.451 & & & \\
\hline Glu11 & 8.252 & 4.157 & 2.315 & $\begin{array}{l}\gamma \mathrm{CH}_{2}: 2.089, \\
2.315\end{array}$ & & -0.133 \\
\hline Lys 12 & 8.063 & 4.232 & $\begin{array}{l}1.913, \\
1.833\end{array}$ & $\begin{array}{l}\gamma \mathrm{CH}_{2}: 1.445 \\
\delta \mathrm{CH}_{2}: 1.687\end{array}$ & & -0.088 \\
\hline Ala13 & 7.962 & 4.287 & 1.415 & & & -0.053 \\
\hline Leu14 & 8.090 & 4.314 & 1.679 & $\begin{array}{l}\gamma \mathrm{CH}_{2}: 1.586 \\
\delta \mathrm{CH}_{3}: 0.888, \\
0.951\end{array}$ & & -0.036 \\
\hline Lys 15 & 8.278 & 4.325 & $\begin{array}{l}1.853, \\
1.793 \\
\end{array}$ & $\begin{array}{l}\gamma \mathrm{CH}_{2}: 1.696 \\
\delta \mathrm{CH}_{2}: 1.461 \\
\end{array}$ & & 0.005 \\
\hline Ser16 & 8.555 & 4.623 & $\begin{array}{l}3.950, \\
4.250\end{array}$ & & & 0.133 \\
\hline Leu17 & 8.353 & 4.317 & 1.700 & $\begin{array}{l}\gamma \mathrm{CH}_{2}: 1.622 \\
\delta \mathrm{CH}_{3}: 0.884, \\
0.949\end{array}$ & & -0.033 \\
\hline
\end{tabular}


Table S10: Chemical shift values (p.p.m) of glycopeptide 11 as measured in a $30 \%$ TFE- $\mathrm{d}_{3} / \mathrm{H}_{2} \mathrm{O}$ mixture at $2.77 \mathrm{mM}$ concentration, $\mathrm{pH}=4.55$ and at $20^{\circ} \mathrm{C}$.

\begin{tabular}{|c|c|c|c|c|c|c|}
\hline Residue & $\mathrm{NH}$ & $\mathrm{C}^{\alpha} \mathrm{H}$ & $\mathrm{C}^{\beta} \mathrm{H}$ & Others & ${ }^{3} \mathrm{~J}_{\alpha \mathrm{NH}} \mathrm{Hz}$ & $\begin{array}{l}\Delta \delta \\
\mathrm{ppm}\end{array}$ \\
\hline DThr2 & 8.421 & 4.203 & n.o & 0.935 & 7.3 & -0.187 \\
\hline Gly3 & 8.402 & $\begin{array}{l}3.910, \\
3.984\end{array}$ & & & Overlap & -0.063 \\
\hline Phe4 & 8.114 & 4.659 & 3.118 & & 6.4 & 0.039 \\
\hline Leu5 & 8.194 & 4.298 & 1.631 & $\begin{array}{l}\gamma \mathrm{CH}_{2}: 1.562 \\
\delta \mathrm{CH}_{3}: 0.882, \\
0.935\end{array}$ & 6.4 & -0.052 \\
\hline Gly6 & 7.683 & 3.900 & & & 11.1 & -0.11 \\
\hline Gly7 & 8.120 & 3.973 & & & 5.61 & -0.037 \\
\hline Asn8 & 8.219 & 4.684 & 2.857 & & 6.69 & -0.076 \\
\hline Leu9 & 8.040 & 4.123 & 1.681 & $\begin{array}{l}\gamma \mathrm{CH}_{2}: 1.681 \\
\delta \mathrm{CH}_{3}: 0.907, \\
0.961\end{array}$ & 5.5 & -0.227 \\
\hline Aib10 & 7.982 & & 1.685 & & - & \\
\hline Glu11 & 7.977 & 3.963 & 2.128 & $\begin{array}{l}\gamma \mathrm{CH}_{2}: 2.388, \\
2.449\end{array}$ & 5.24 & -0.327 \\
\hline Lys 12 & 7.874 & 4.079 & 1.995 & $\begin{array}{l}\gamma \mathrm{CH}_{2}: 1.507 \\
\delta \mathrm{CH}_{2}: 1.635, \\
1.720\end{array}$ & 5.6 & -0.241 \\
\hline Ala13 & 8.008 & 4.249 & 1.507 & & 5.7 & -0.091 \\
\hline Leu14 & 8.080 & 4.228 & 1.834 & $\begin{array}{l}\gamma \mathrm{CH}_{2}: 1.587 \\
\delta \mathrm{CH}_{3}: 0.899\end{array}$ & 5.58 & -0.122 \\
\hline Lys 15 & 7.915 & 4.216 & 1.971 & $\begin{array}{l}\gamma \mathrm{CH}_{2}: 1.524 \\
\delta \mathrm{CH}_{2}: 1.608, \\
1.746\end{array}$ & 5.17 & -0.104 \\
\hline Ser16 & 8.017 & 4.616 & $\begin{array}{l}4.041, \\
4.332\end{array}$ & & 6.5 & 0.126 \\
\hline Leu17 & 7.987 & 4.312 & 1.819 & $\begin{array}{l}\gamma \mathrm{CH}_{2}: 1.654 \\
\delta \mathrm{CH}_{3}: 0.911, \\
0.966\end{array}$ & 7.07 & -0.038 \\
\hline
\end{tabular}


Table S11: Chemical shift values (p.p.m) of glycopeptide 11 as measured in SDS at $3.16 \mathrm{mM}$ concentration, $\mathrm{pH}=4.5$ and at $25^{\circ} \mathrm{C}$.

\begin{tabular}{|c|c|c|c|c|c|}
\hline Residue & $\mathrm{NH}$ & $\mathrm{C}^{\alpha} \mathrm{H}$ & $\mathrm{C}^{\beta} \mathrm{H}$ & Others & $\begin{array}{l}\Delta \delta \\
\mathrm{ppm}\end{array}$ \\
\hline Tyr1 & - & 4.437 & $3.034,3.217$ & 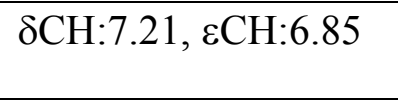 & $\begin{array}{l}- \\
0.123\end{array}$ \\
\hline DThr2 & 8.647 & 4.364 & 4.364 & $\gamma \mathrm{CH}_{3}: 1.156$ & $-\overline{0}$ \\
\hline Gly3 & 8.450 & 3.928 & & & $\begin{array}{l}- \\
0.082\end{array}$ \\
\hline Phe4 & 7.974 & 4.495 & $3.013,3.181$ & $\begin{array}{l}\delta \mathrm{CH}: 6.863, \varepsilon \mathrm{CH}: 7.27, \\
\zeta \mathrm{CH}: 7.211\end{array}$ & $-\overline{0}$ \\
\hline Leu5 & 7.869 & 4.303 & 1.805 & $\begin{array}{l}\gamma \mathrm{CH}_{2}: 1.549 \\
\delta \mathrm{CH}_{3}: 0.881,0.950 \\
\end{array}$ & $\begin{array}{l}- \\
0.047 \\
\end{array}$ \\
\hline Gly6 & 7.994 & $3.899,4.002$ & & & $\begin{array}{l}- \\
0.060\end{array}$ \\
\hline Gly7 & 8.183 & 4.029 & & & 0.019 \\
\hline Asn8 & 8.130 & 4.516 & 2.801 & $\delta \mathrm{NH}: 7.50,6.838$ & $\begin{array}{l}- \\
0.244 \\
\end{array}$ \\
\hline Leu9 & 8.185 & 4.029 & 1.812 & $\begin{array}{l}\gamma \mathrm{CH}_{2}: 1.611 \\
\delta \mathrm{CH}_{3}: 0.898,0.970\end{array}$ & $\begin{array}{l}- \\
0.321\end{array}$ \\
\hline Aib10 & 8.252 & - & $1.379,1.423$ & & \\
\hline Glu11 & 7.846 & 3.963 & 2.127 & $\gamma \mathrm{CH}_{2}: 2.531$ & $\begin{array}{ll}- \\
0.327\end{array}$ \\
\hline Lys 12 & 7.825 & 4.007 & 2.008 & $\begin{array}{l}\gamma \mathrm{CH}_{2}: 1.678 \\
\delta \mathrm{CH}_{2}: 1.906\end{array}$ & $\begin{array}{l}- \\
0.313\end{array}$ \\
\hline Ala13 & 7.979 & 4.160 & 1.432 & & -0.18 \\
\hline Leu14 & 8.012 & 4.105 & 1.773 & $\begin{array}{l}\gamma \mathrm{CH}_{2}: 1.611 \\
\delta \mathrm{CH}_{3}: 0.892\end{array}$ & $\begin{array}{l}- \\
0.245\end{array}$ \\
\hline Lys 15 & 7.866 & 4.112 & 1.906 & $\begin{array}{l}\gamma \mathrm{CH}_{2}: 1.549 \\
\delta \mathrm{CH}_{2}: 1.681,1.805\end{array}$ & $\begin{array}{l}- \\
0.208\end{array}$ \\
\hline Ser16 & 7.928 & 4.610 & $4.088,4.155$ & & 0.12 \\
\hline Leu17 & 7.894 & 4.222 & 1.771 & $\begin{array}{l}\gamma \mathrm{CH}_{2}: 1.584 \\
\delta \mathrm{CH}_{3}: 0.888\end{array}$ & $\begin{array}{l}-128 \\
0.128\end{array}$ \\
\hline $\mathrm{CONH}_{2}$ & $\begin{array}{l}6.910, \\
7.256\end{array}$ & & & & \\
\hline
\end{tabular}


Table S12: Chemical shift values (p.p.m) of glycopeptide $\mathbf{1 2}$ as measured in H2O:D2O (9:1) at $2.47 \mathrm{mM}$ concentration, $\mathrm{pH}=4.5$ and at $15^{\circ} \mathrm{C}$.

\begin{tabular}{|c|c|c|c|c|c|}
\hline Residue & $\mathrm{NH}$ & $\mathrm{C}^{\alpha} \mathrm{H}$ & $\mathrm{C}^{\beta} \mathrm{H}$ & Others & $\begin{array}{l}\Delta \delta \text { (Obs.-r.c.) } \\
\text { ppm }\end{array}$ \\
\hline Tyr1 & & 4.327 & $\begin{array}{l}3.128, \\
3.186\end{array}$ & $\begin{array}{l}\delta \mathrm{CH}: 7.17,7.187, \\
\varepsilon \mathrm{CH}: 6.901\end{array}$ & -0.233 \\
\hline DThr2 & 8.579 & 4.164 & 4.099 & $\gamma \mathrm{CH}_{2}: 0.890$ & -0.226 \\
\hline Gly3 & 8.462 & 3.881 & & & -0.129 \\
\hline Phe4 & 8.128 & 4.623 & $\begin{array}{l}3.018 \\
3.076\end{array}$ & $\begin{array}{l}\delta \mathrm{CH}: 7.24,7.253 \\
\varepsilon \mathrm{CH}: 7.443, \zeta \mathrm{CH}: 7.443\end{array}$ & -0.003 \\
\hline Leu5 & 8.291 & 4.612 & 1.582 & $\begin{array}{l}\gamma \mathrm{CH}_{2}: 1.532 \\
\delta \mathrm{CH}_{3}: 0.919\end{array}$ & 0.262 \\
\hline Pro6 & & 4.329 & & $\begin{array}{l}\gamma \mathrm{CH}_{2}: \\
\delta \mathrm{CH}_{2}:\end{array}$ & -0.111 \\
\hline Asn7 & 8.539 & 4.648 & $\begin{array}{l}2.797, \\
2.851\end{array}$ & $\delta \mathrm{NH}: 6.976,7.671$ & -0.112 \\
\hline Leu8 & 8.249 & 4.213 & 1.590 & $\begin{array}{l}\gamma \mathrm{CH}_{2}: 1.590 \\
\delta \mathrm{CH}_{3}: 0.866,0.941\end{array}$ & -0.128 \\
\hline Aib9 & 8.269 & & 1.455 & & \\
\hline Glu10 & 8.233 & 4.177 & 2.351 & $\gamma \mathrm{CH}_{2}: 2.032,2.093$ & -0.113 \\
\hline Lys11 & 8.080 & 4.233 & $\begin{array}{l}1.830, \\
1.909\end{array}$ & $\begin{array}{l}\gamma \mathrm{CH}_{2}: 1.441,1.529 \\
\delta \mathrm{CH}_{2}: 1.693\end{array}$ & -0.087 \\
\hline Ala12 & 7.982 & 4.284 & 1.410 & & -0.056 \\
\hline Leu13 & 8.098 & 4.314 & 1.679 & $\begin{array}{l}\gamma \mathrm{CH}_{2}: 1.586 \\
\delta \mathrm{CH}_{3}: 0.890,0.945\end{array}$ & -0.036 \\
\hline Lys 14 & 8.285 & 4.331 & 1.852 & $\begin{array}{l}\gamma \mathrm{CH}_{2}: 1.697 \\
\delta \mathrm{CH}_{2}: 1.794\end{array}$ & 0.011 \\
\hline Ser15 & 8.560 & 4.627 & $\begin{array}{l}3.951, \\
4.251 \\
\end{array}$ & & 0.137 \\
\hline Leu16 & 8.349 & 4.324 & 1.707 & $\begin{array}{l}\gamma \mathrm{CH}_{2}: 1.626 \\
\delta \mathrm{CH}_{3}: 0.884,0.947 \\
\end{array}$ & -0.026 \\
\hline$-\mathrm{CONH}_{2}$ & 7.443 & & & & \\
\hline
\end{tabular}


Table S13: Chemical shift values (p.p.m) of glycopeptide 12 as measured in a $30 \%$ TFE- $\mathrm{d}_{3} / \mathrm{H}_{2} \mathrm{O}$ mixture at $2.75 \mathrm{mM}$ concentration, $\mathrm{pH}=4.5$ and at $20^{\circ} \mathrm{C}$.

\begin{tabular}{|c|c|c|c|c|c|}
\hline Residue & $\mathrm{NH}$ & $\mathrm{C}^{\alpha} \mathrm{H}$ & $\mathrm{C}^{\beta} \mathrm{H}$ & Others & $\begin{array}{l}\Delta \delta \text { (Obs.-r.c.) } \\
\text { ppm }\end{array}$ \\
\hline Tyr1 & & 4.356 & $\begin{array}{l}3.155 \\
3.214\end{array}$ & $\begin{array}{l}\delta \mathrm{CH}: 7.207,7.220 \\
\varepsilon \mathrm{CH}: 6.920\end{array}$ & -0.204 \\
\hline Thr2 & 8.391 & 4.214 & 4.179 & $\gamma \mathrm{CH}_{3}: 0.930$ & -0.176 \\
\hline Gly3 & 8.332 & $\begin{array}{l}3.924, \\
3.968\end{array}$ & & & -0.064 \\
\hline Phe4 & 8.018 & 4.670 & 3.104 & $\begin{array}{l}\delta \mathrm{CH}: 7.02, \varepsilon \mathrm{CH}: 7.441, \\
\zeta \mathrm{CH}: 7.262,7.275\end{array}$ & 0.05 \\
\hline Leu5 & 7.893 & 4.559 & 1.669 & $\begin{array}{l}\gamma \mathrm{CH}_{2}: 1.620 \\
\delta \mathrm{CH}_{3}: 0.959\end{array}$ & 0.209 \\
\hline Pro6 & & 4.298 & $2.06,2.32$ & $\begin{array}{l}\gamma \mathrm{CH}_{2}: 1.88,1.99 \\
\delta \mathrm{CH}_{2}: 3.542,3.668\end{array}$ & -0.142 \\
\hline Asn7 & 8.108 & 4.573 & 2.844 & $\delta \mathrm{NH}: 7.60,6.82$ & -0.187 \\
\hline Leu8 & 7.818 & 4.150 & 1.680 & $\begin{array}{l}\gamma \mathrm{CH}_{2}: 1.680 \\
\delta \mathrm{CH}_{3}: 0.910,0.964\end{array}$ & -0.2 \\
\hline Aib9 & 8.046 & & 1.452 & & \\
\hline Glu10 & 8.010 & 3.946 & 2.141 & $\gamma \mathrm{CH}_{2}: 2.392,2.474$ & -0.344 \\
\hline Lys11 & 7.799 & 4.068 & 2.012 & $\begin{array}{l}\gamma \mathrm{CH}_{2}: 1.729 \\
\delta \mathrm{CH}_{2}: 1.729\end{array}$ & -0.252 \\
\hline Ala12 & 8.005 & 4.239 & 1.507 & & -0.101 \\
\hline Leu13 & 8.149 & 4.210 & 1.843 & $\begin{array}{l}\gamma \mathrm{CH}_{2}: 1.578 \\
\delta \mathrm{CH}_{3}: 0.896\end{array}$ & -0.14 \\
\hline Lys 14 & 7.936 & 4.201 & 1.979 & $\begin{array}{l}\gamma \mathrm{CH}_{2}: 1.752 \\
\delta \mathrm{CH}_{2}: 1.752\end{array}$ & -0.119 \\
\hline Ser15 & 7.975 & 4.631 & $\begin{array}{l}4.072, \\
4.335\end{array}$ & & 0.141 \\
\hline Leu16 & 7.983 & 4.306 & 1.830 & $\begin{array}{l}\gamma \mathrm{CH}_{2}: 1.656 \\
\delta \mathrm{CH}_{3}: 0.915,0.963\end{array}$ & -0.044 \\
\hline$-\mathrm{CONH}_{2}$ & 7.270 & & & & \\
\hline
\end{tabular}


Table S14: Chemical shift values (p.p.m) of glycopeptide 12 as measured in SDS at $2.3 \mathrm{mM}$ concentration, $\mathrm{pH}=4.5$ and at $25^{\circ} \mathrm{C}$.

\begin{tabular}{|l|l|l|l|l|l|}
\hline Residue & $\mathrm{NH}$ & $\mathrm{C}^{\alpha} \mathrm{H}$ & $\mathrm{C}^{\beta} \mathrm{H}$ & Others & $\begin{array}{l}\Delta \delta \text { (Obs.-R.C.) } \\
\text { ppm }\end{array}$ \\
\hline Tyr1 & & 4.48 & $3.085,3.284$ & $\delta \mathrm{CH}: 7.253, \varepsilon \mathrm{CH}: 6.889$ & -0.08 \\
\hline Thr2 & 8.481 & 4.380 & s.o. & $\gamma \mathrm{CH}_{3}: 1.21$ & -0.01 \\
\hline Gly3 & 8.477 & 3.964 & & & -0.055 \\
\hline Phe4 & 7.868 & 4.635 & $3.098,3.227$ & $\begin{array}{l}\delta \mathrm{CH}: 6.99, \varepsilon \mathrm{CH}: 7.33, \\
\zeta \mathrm{CH}: 7.28\end{array}$ & 0.015 \\
\hline Leu5 & 8.064 & 4.391 & 1.895 & $\begin{array}{l}\gamma \mathrm{CH}_{2}: 1.610,1.670 \\
\delta \mathrm{CH}_{3}: 0.972,1.029\end{array}$ & 0.041 \\
\hline Pro6 & & 4.32 & $2.05,2.32$ & $\begin{array}{l}\gamma \mathrm{CH}_{2}: 1.87,1.977 \\
\delta \mathrm{CH}_{2}: 3.63,3.58\end{array}$ & -0.12 \\
\hline Asn7 & 7.792 & 4.609 & 2.885 & $\delta \mathrm{NH}_{2}: 583,6.909$ & -0.151 \\
\hline Leu8 & 7.858 & 4.034 & 1.712 & $\begin{array}{l}\gamma \mathrm{CH}_{2}: 1.712 \\
\delta \mathrm{CH}_{3}: 0.94,0.979\end{array}$ & -0.316 \\
\hline Aib9 & 8.399 & - & 1.530 & & -0.277 \\
\hline Glu10 & 7.688 & 4.013 & 2.136 & $\gamma \mathrm{CH}_{2}: 2.291,2.399$ & -0.235 \\
\hline Lys11 & 7.831 & 4.085 & 2.028 & $\begin{array}{l}\gamma \mathrm{CH}_{2}: 1.944,1.708 \\
\delta \mathrm{CH}_{2}: 1.528\end{array}$ & -0.111 \\
\hline Ala12 & 8.122 & 4.229 & 1.472 & & -0.168 \\
\hline Leu13 & 8.104 & 4.182 & 1.854 & $\begin{array}{l}\gamma \mathrm{CH}_{2}: 1.642 \\
\delta \mathrm{CH}_{3}: 0.944\end{array}$ & -0.122 \\
\hline Lys14 & 7.879 & 4.198 & 1.944 & $\begin{array}{l}\gamma \mathrm{CH}_{2}: 1.503,1.587 \\
\delta \mathrm{CH}_{2}: 1.743\end{array}$ & -0.063 \\
\hline Ser15 & 8.001 & 4.700 & 4.162 & $\begin{array}{l}\gamma \mathrm{CH}_{2}: 1.649 \\
\delta \mathrm{CH}_{3}: 0.942\end{array}$ & \\
\hline Leu16 & 7.983 & 4.287 & 1.800 & & 0.21 \\
\hline -CONH & 6.970, & & & & \\
\hline
\end{tabular}


Table S15: Chemical shift values (p.p.m) of peptide $9 \mathbf{u}$ as measured in SDS at $2.5 \mathrm{mM}$ concentration, $\mathrm{pH}=4.5$ and at $25^{\circ} \mathrm{C}$.

\begin{tabular}{|c|c|c|c|c|c|}
\hline Residue & $\mathrm{NH}$ & $\mathrm{C}^{\alpha} \mathrm{H}$ & $\mathrm{C}^{\beta} \mathrm{H}$ & others & $\begin{array}{l}\Delta \delta \text { (obs-r.c) } \\
\text { ppm }\end{array}$ \\
\hline Tyr1 & & 4.455 & $3.049,3.239$ & $\delta \mathrm{CH}: 7.22, \varepsilon \mathrm{CH}: 6.86$ & -0.105 \\
\hline DThr2 & 8.645 & 4.350 & & $\gamma \mathrm{CH}_{3}: 1.164$ & -0.04 \\
\hline Gly3 & 8.445 & 3.945 & & & -0.065 \\
\hline Phe4 & 7.884 & 4.593 & $3.055,3.216$ & $\begin{array}{l}\delta \mathrm{CH}: 6.86, \varepsilon \mathrm{CH}: 7.28, \\
\zeta \mathrm{CH}: 7.22\end{array}$ & -0.027 \\
\hline Leu5 & 8.114 & 4.303 & 1.876 & $\begin{array}{l}\gamma \mathrm{CH}_{2}: 1.569 \\
\delta \mathrm{CH}_{3}: 0.98\end{array}$ & -0.047 \\
\hline Pro6 & & 4.24 & $2.10,2.31$ & $\begin{array}{l}\gamma \mathrm{CH}_{2}: 1.95 \\
\delta \mathrm{CH}_{2}: 3.615 \\
\end{array}$ & \\
\hline Asn7 & 7.661 & 4.526 & $2.795,2.870$ & $\delta \mathrm{NH}: 6.879,7.56$ & -0.234 \\
\hline Leu8 & 7.861 & 3.975 & 1.650 & $\begin{array}{l}\gamma \mathrm{CH}_{2}: 1.650 \\
\delta \mathrm{CH}_{3}: 0.901,0.940\end{array}$ & -0.375 \\
\hline Aib9 & 8.466 & - & $1.404,1.495$ & & \\
\hline Glu10 & 7.742 & 3.954 & 2.122 & $\gamma \mathrm{CH}_{2}: 2.363,2.465$ & -0.336 \\
\hline Lys11 & 7.817 & 4.024 & $1.933,2.001$ & $\begin{array}{l}\gamma \mathrm{CH}_{2}: 1.486 \\
\delta \mathrm{CH}_{2}: 1.644 \\
\end{array}$ & -0.296 \\
\hline Ala12 & 8.192 & 4.098 & 1.462 & & -0.242 \\
\hline Leu13 & 8.176 & 4.054 & 1.813 & $\begin{array}{l}\gamma \mathrm{CH}_{2}: 1.626 \\
\delta \mathrm{CH}_{3}: 0.908 \\
\end{array}$ & -0.296 \\
\hline Lys 14 & 8.025 & 4.117 & 1.926 & $\begin{array}{l}\gamma \mathrm{CH}_{2}: 1.498,1.580 \\
\delta \mathrm{CH}_{2}: 1.687, \varepsilon \mathrm{CH}_{2}: 2.990\end{array}$ & -0.203 \\
\hline Ser15 & 7.772 & 4.365 & 4.256 & & -0.125 \\
\hline Leu16 & 7.769 & 3.953 & 1.795 & $\begin{array}{l}\gamma \mathrm{CH}_{2}: 1.585 \\
\delta \mathrm{CH}_{3}: 0.898\end{array}$ & -0.397 \\
\hline$-\mathrm{CONH}_{2}$ & $\begin{array}{l}6.92, \\
7.18\end{array}$ & & & & \\
\hline
\end{tabular}




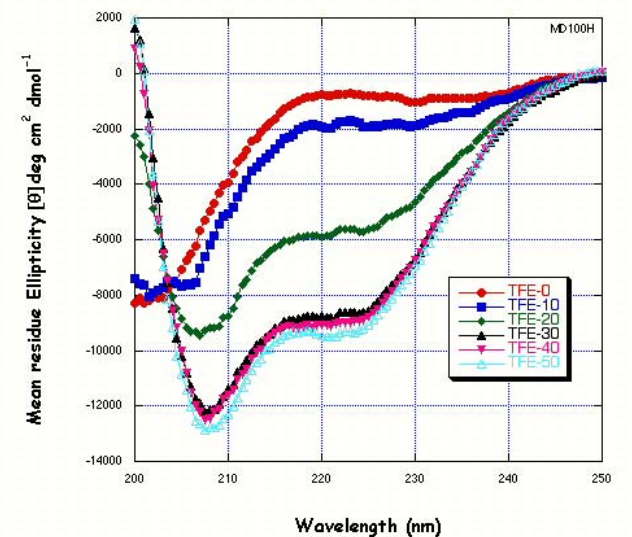

a)

Wovelength (nm)

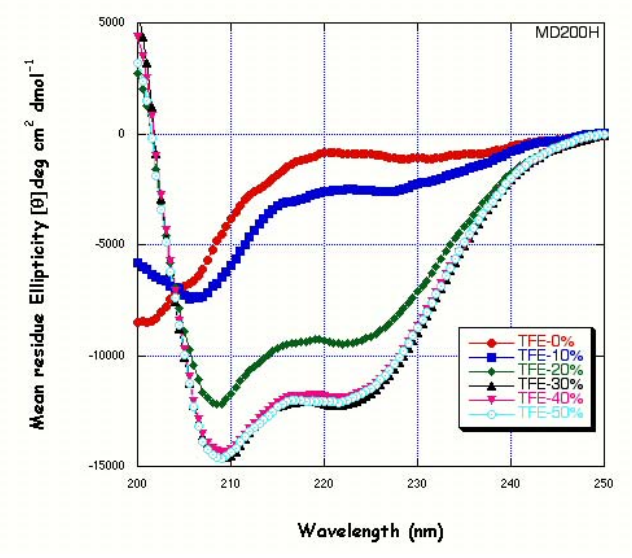

Figure S1: Far-UV CD spectra of a) glycopeptide 9 and b) peptide $\mathbf{9 u}$ as a function of TFE concentration.

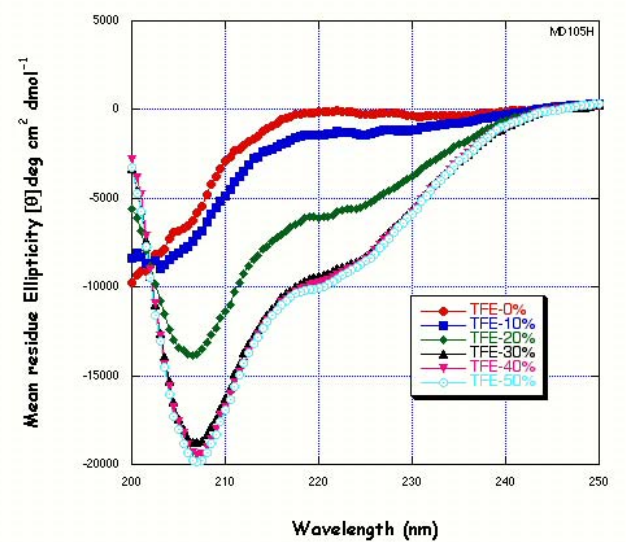

a)

Wovelength (nn)

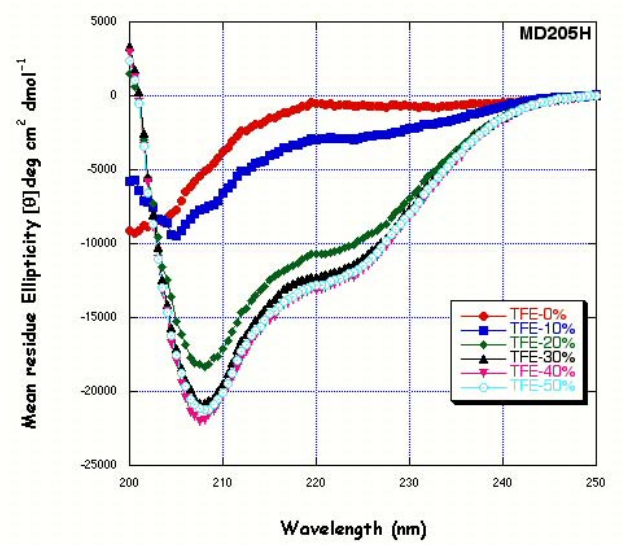

Figure S2: Far-UV CD spectra of a)glycopeptide $\mathbf{1 0}$ and b) peptide $\mathbf{1 0 u}$ as a function of TFE concentration. 


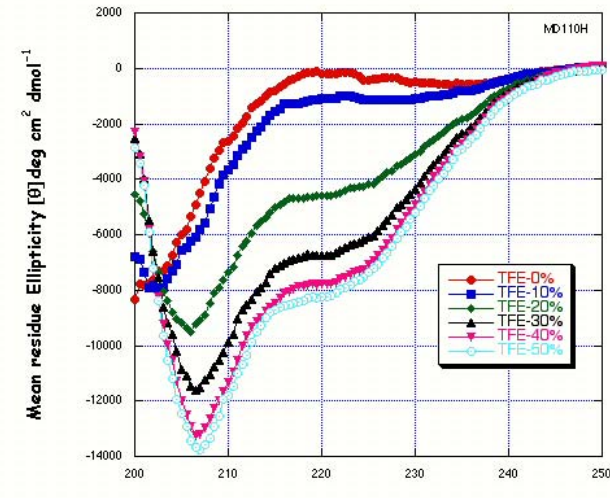

a)

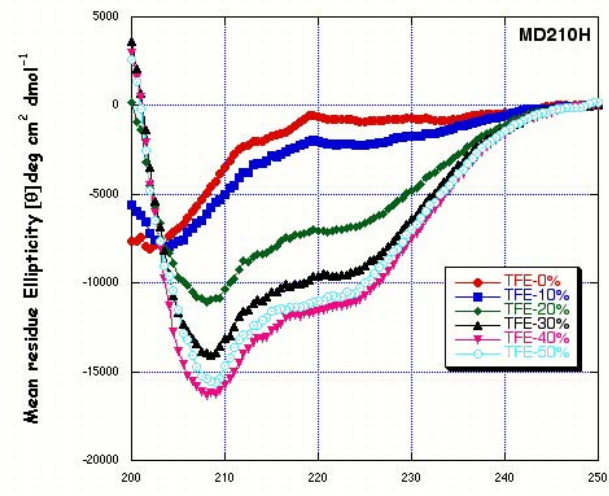

b)

Wovelength (nm)

Figure S3: Far-UV CD spectra of a) glycopeptide 11 and b) peptide $\mathbf{1 1} \mathbf{u}$ as a function of TFE concentration.

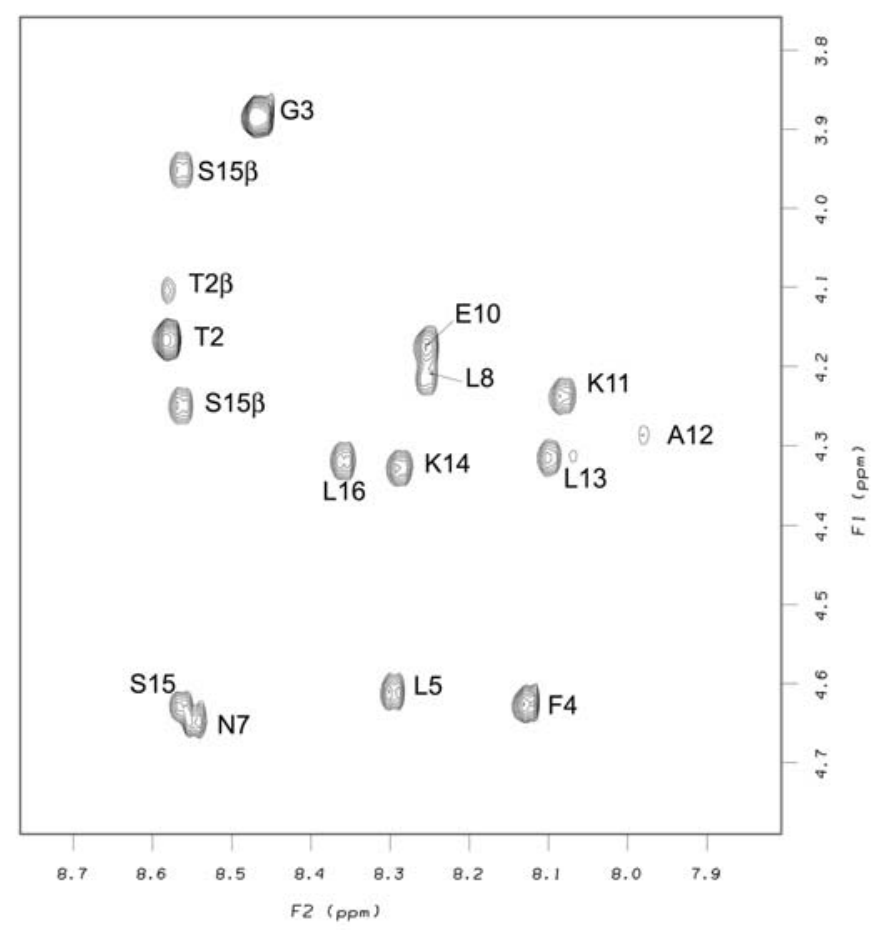

Figure S4: Finger print $(\alpha \mathrm{H}-\mathrm{NH})$ region of TOCSY (mixing time $=100 \mathrm{~ms}$ ) spectra of glycopeptide 9 in $\mathrm{H}_{2} \mathrm{O}: \mathrm{D}_{2} \mathrm{O}(9: 1)$ at $\mathrm{pH}=4.5$ at $20^{\circ} \mathrm{C}$. 


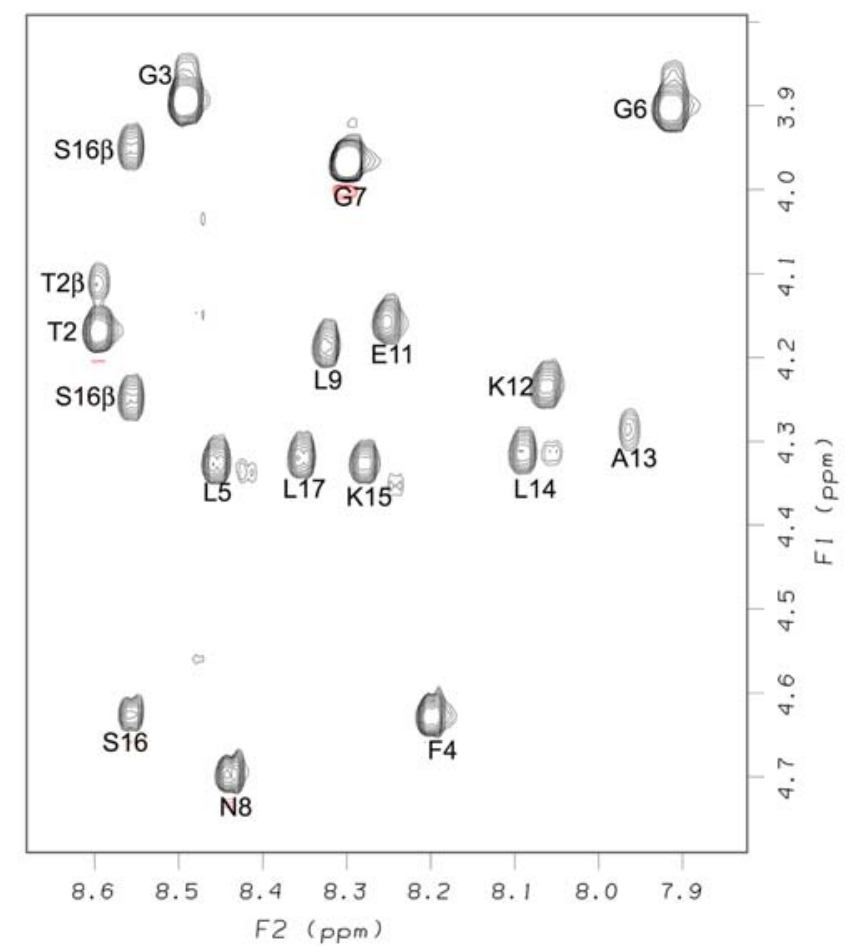

Figure S5: Finger print $(\alpha \mathrm{H}-\mathrm{NH})$ region of TOCSY (mixing time $=100 \mathrm{~ms}$ ) spectra of glycopeptide 11 in $\mathrm{H}_{2} \mathrm{O}: \mathrm{D}_{2} \mathrm{O}(9: 1)$ at $\mathrm{pH}=4.5$ at $20^{\circ} \mathrm{C}$.

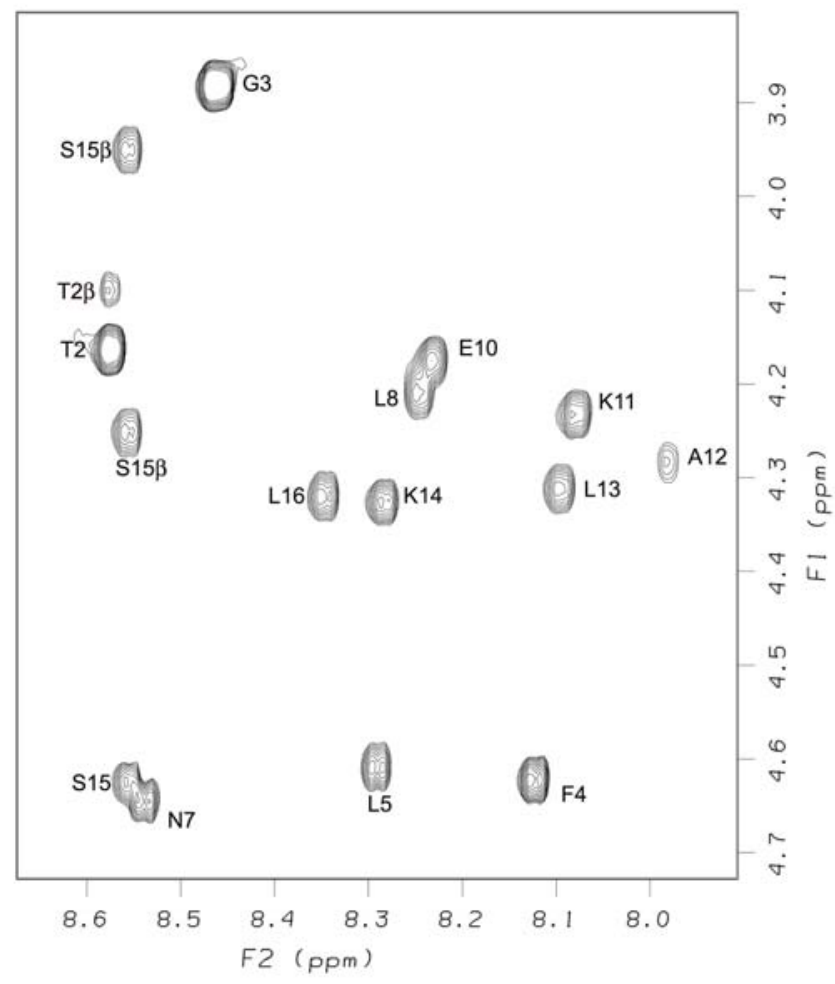

Figure S6: Finger print $(\alpha \mathrm{H}-\mathrm{NH})$ region of TOCSY (mixing time $=100 \mathrm{~ms}$ ) spectra of glycopeptide 12 in $\mathrm{H}_{2} \mathrm{O}: \mathrm{D}_{2} \mathrm{O}(9: 1)$ at $\mathrm{pH}=4.5$ at $20^{\circ} \mathrm{C}$. 


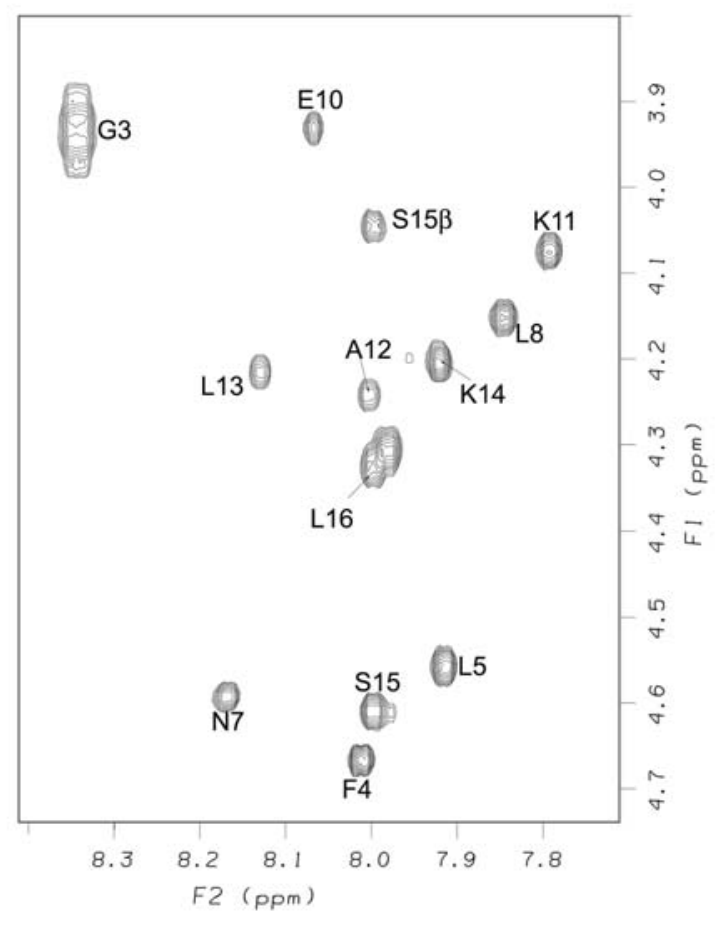

Figure S7. Finger print $(\alpha \mathrm{H}-\mathrm{NH})$ region of TOCSY (mixing time $=100 \mathrm{~ms})$ spectra of glycopeptide 9 in TFE: water (3:7) at $\mathrm{pH}=4.5$ and at $15^{\circ} \mathrm{C}$.

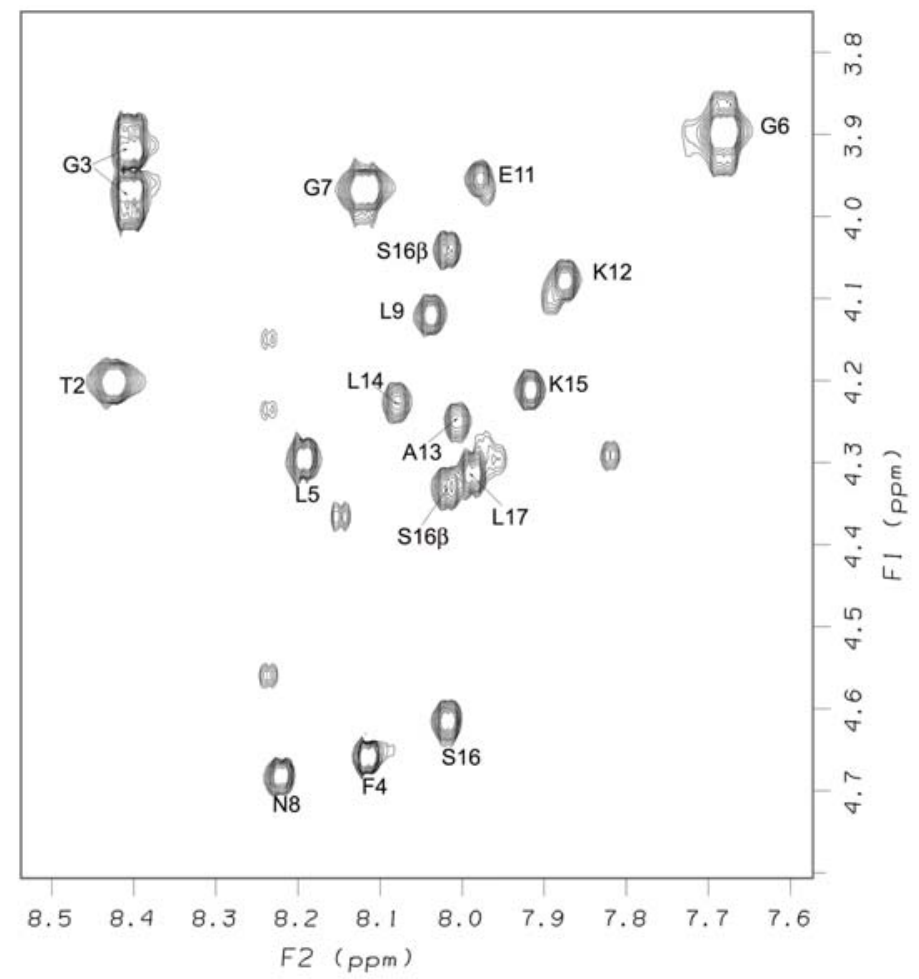

Figure S8. Finger print $(\alpha \mathrm{H}-\mathrm{NH})$ region of TOCSY (mixing time $=100 \mathrm{~ms}$ ) spectra of glycopeptide 11 in TFE:water $(3: 7)$ at $\mathrm{pH}=4.5$ and at $15^{\circ} \mathrm{C}$. 


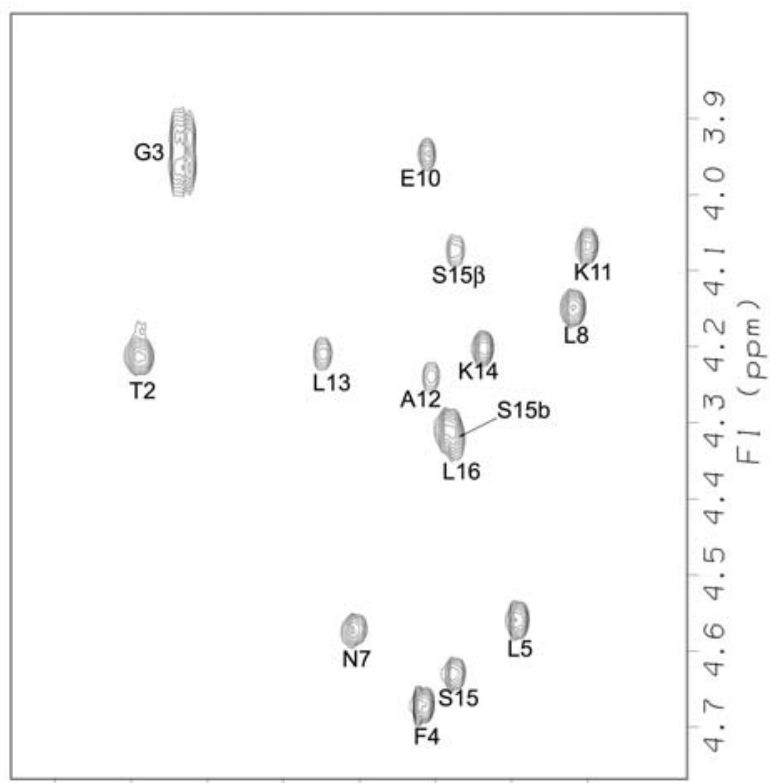

8.58 .48 .38 .28 .18 .07 .97 .8

F2 (ppm)

Figure S9. Finger print $(\alpha \mathrm{H}-\mathrm{NH})$ region of TOCSY (mixing time $=100 \mathrm{~ms}$ ) spectra of glycopeptide 12 in TFE:water $(3: 7)$ at $\mathrm{pH}=4.5$ and at $15^{\circ} \mathrm{C}$.

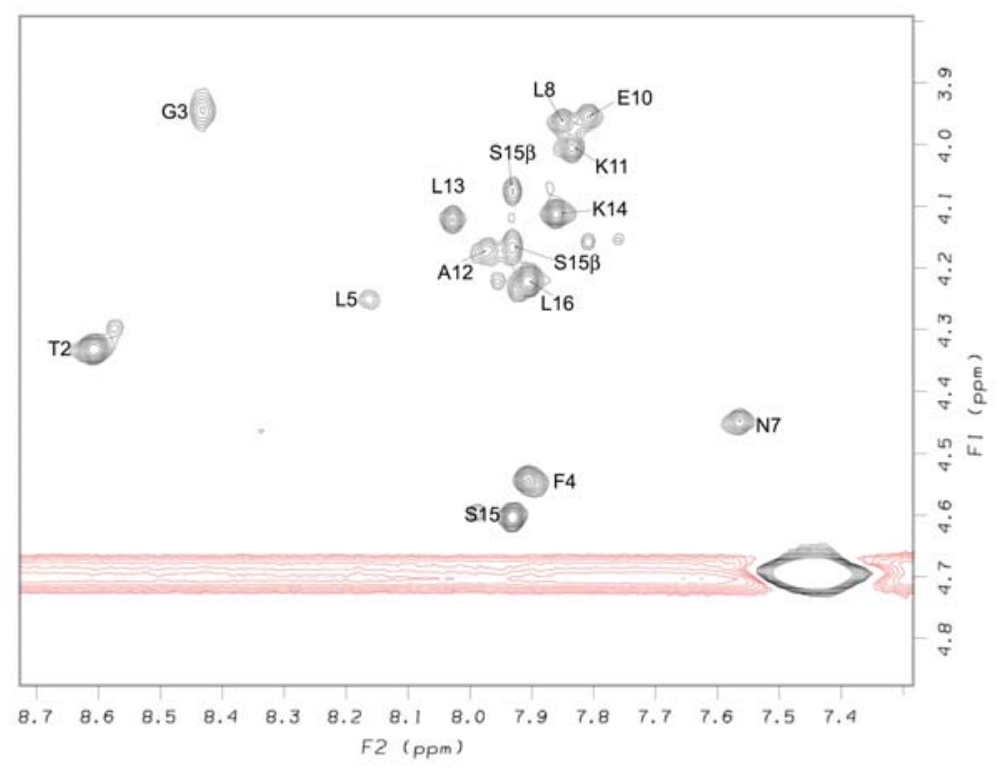

Figure S10. Finger print region of $(\alpha \mathrm{H}-\mathrm{NH})$ of TOCSY (mixing time $=100 \mathrm{~ms})$ spectra of glycopeptide 9 in SDS micelle at $\mathrm{pH}=4.5$ and at $25^{\circ} \mathrm{C}$. 


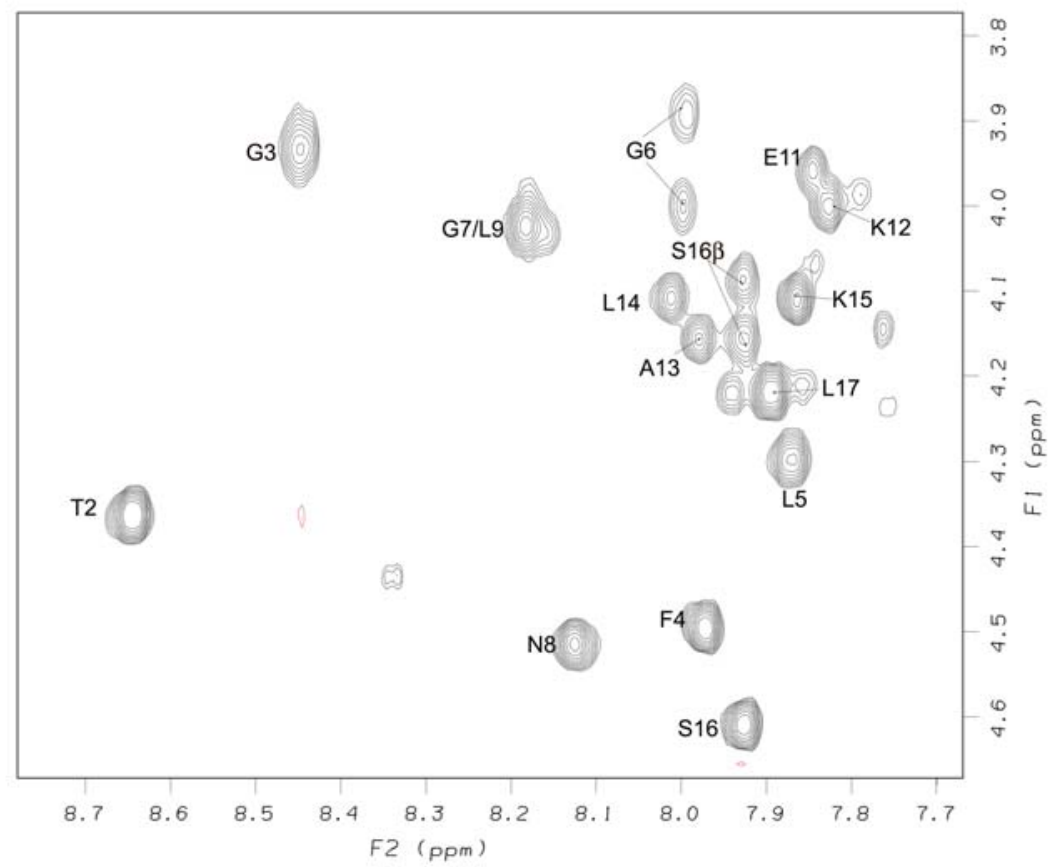

Figure S11. Finger print region of $(\alpha \mathrm{H}-\mathrm{NH})$ of TOCSY (mixing time $=100 \mathrm{~ms})$ spectra of glycopeptide 11 in SDS micelle at $\mathrm{pH}=4.5$ and at $25^{\circ} \mathrm{C}$.

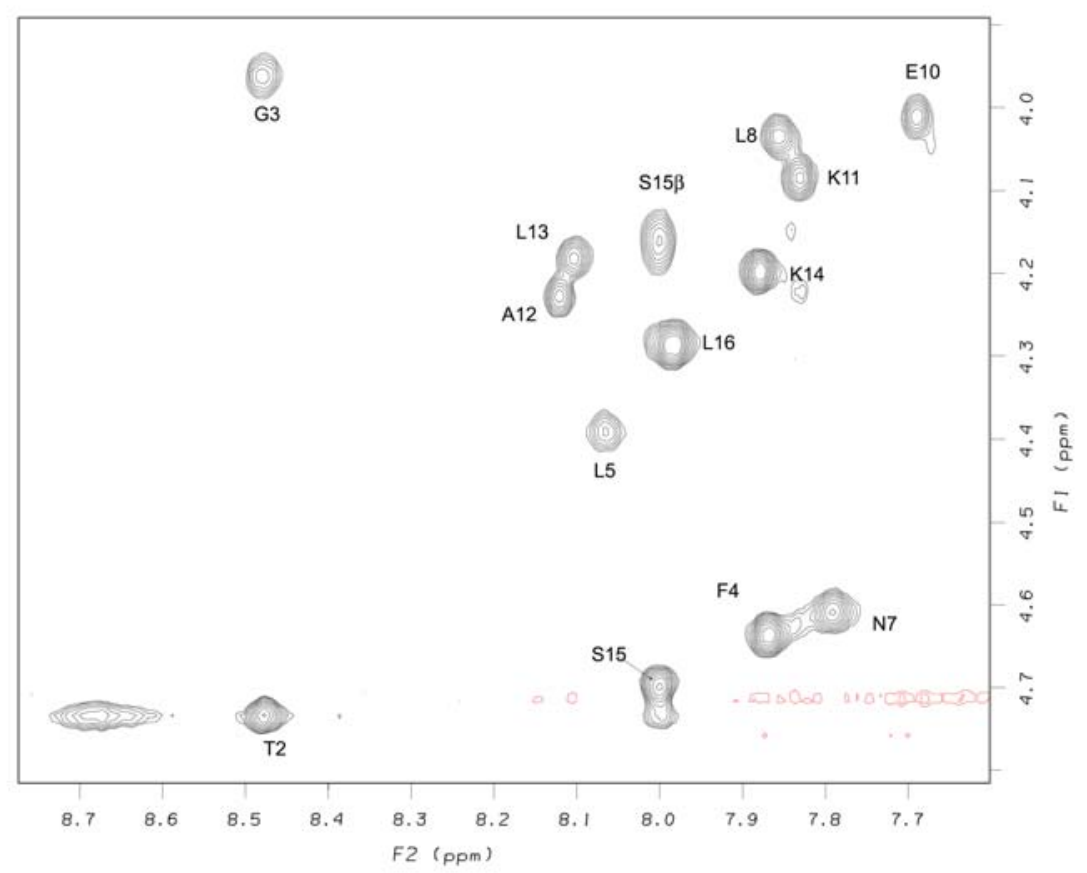

Figure S12. Finger print region of $(\alpha \mathrm{H}-\mathrm{NH})$ of TOCSY (mixing time $=100 \mathrm{~ms})$ spectra of glycopeptide 12 in SDS micelle at $\mathrm{pH}=4.5$ and at $25^{\circ} \mathrm{C}$. 


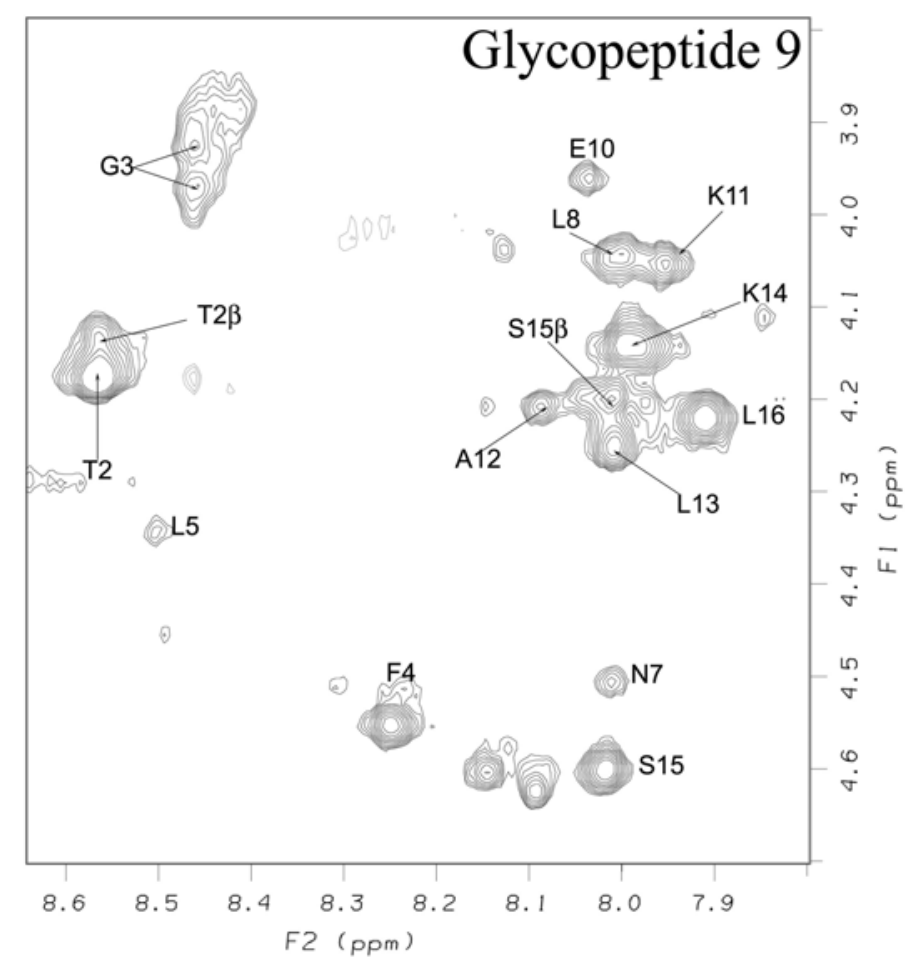

Figure S13: Finger print $(\alpha \mathrm{H}-\mathrm{NH})$ region of TOCSY (mixing time $=80 \mathrm{~ms}$ ) spectra in zwitterionic bicelle at $\mathrm{pH}=4.5$ and at $25^{\circ} \mathrm{C}$.

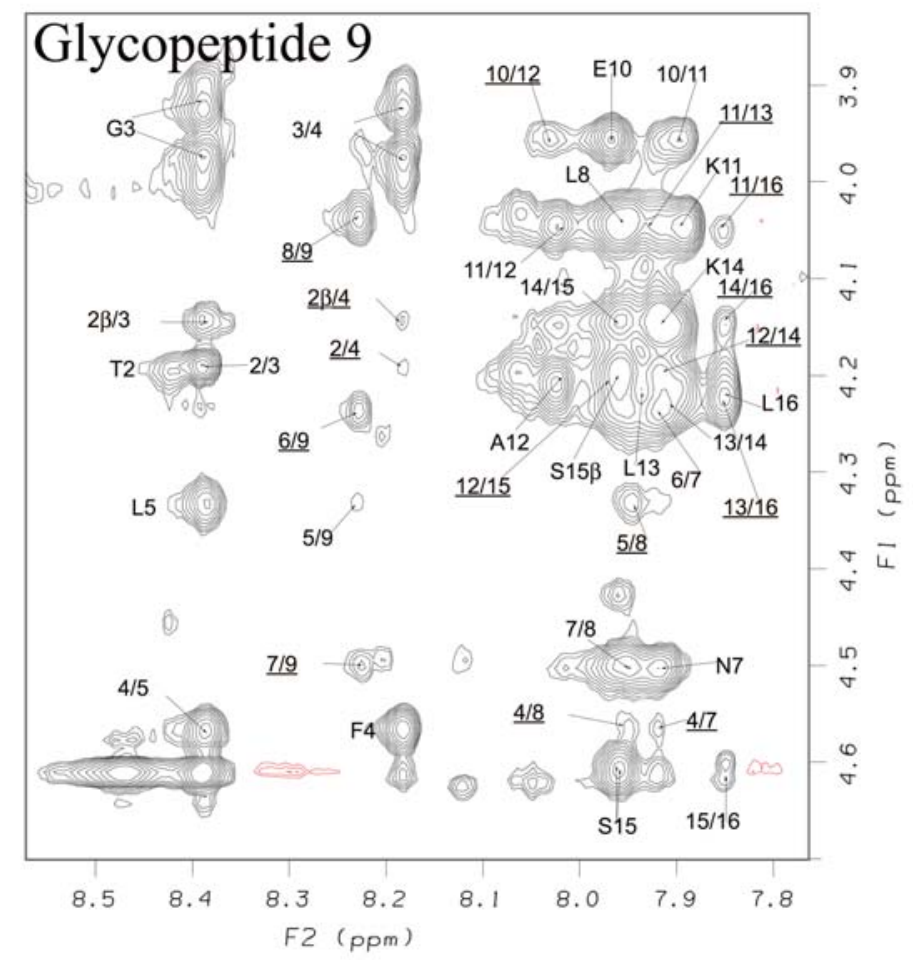

Figure S14: Finger print $(\alpha \mathrm{H}-\mathrm{NH})$ region of NOESY (mixing time $=300 \mathrm{~ms}$ ) spectra in zwitterionic bicelle at $\mathrm{pH}=4.5$ and $38^{\circ} \mathrm{C}$. 


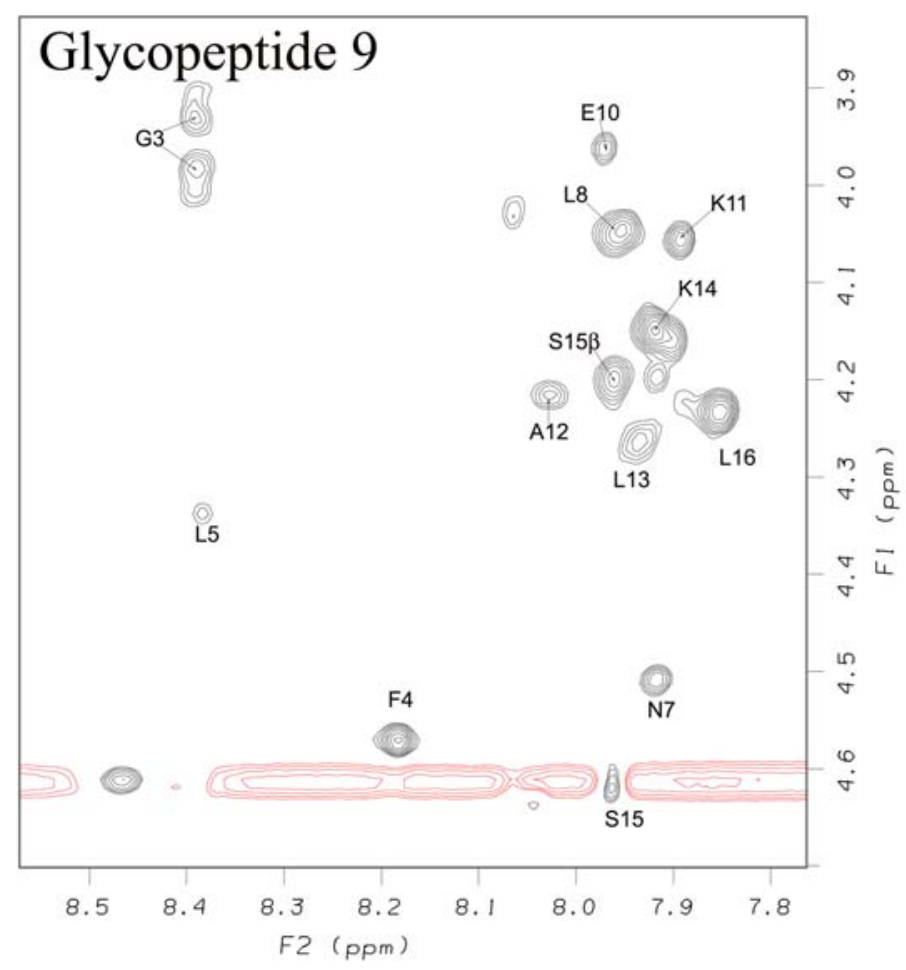

Figure S15: Finger print $(\alpha \mathrm{H}-\mathrm{NH})$ region of TOCSY (mixing time $=80 \mathrm{~ms}$ ) spectra in zwitterionic bicelle at $\mathrm{pH}=4.5$ and $38^{\circ} \mathrm{C}$.

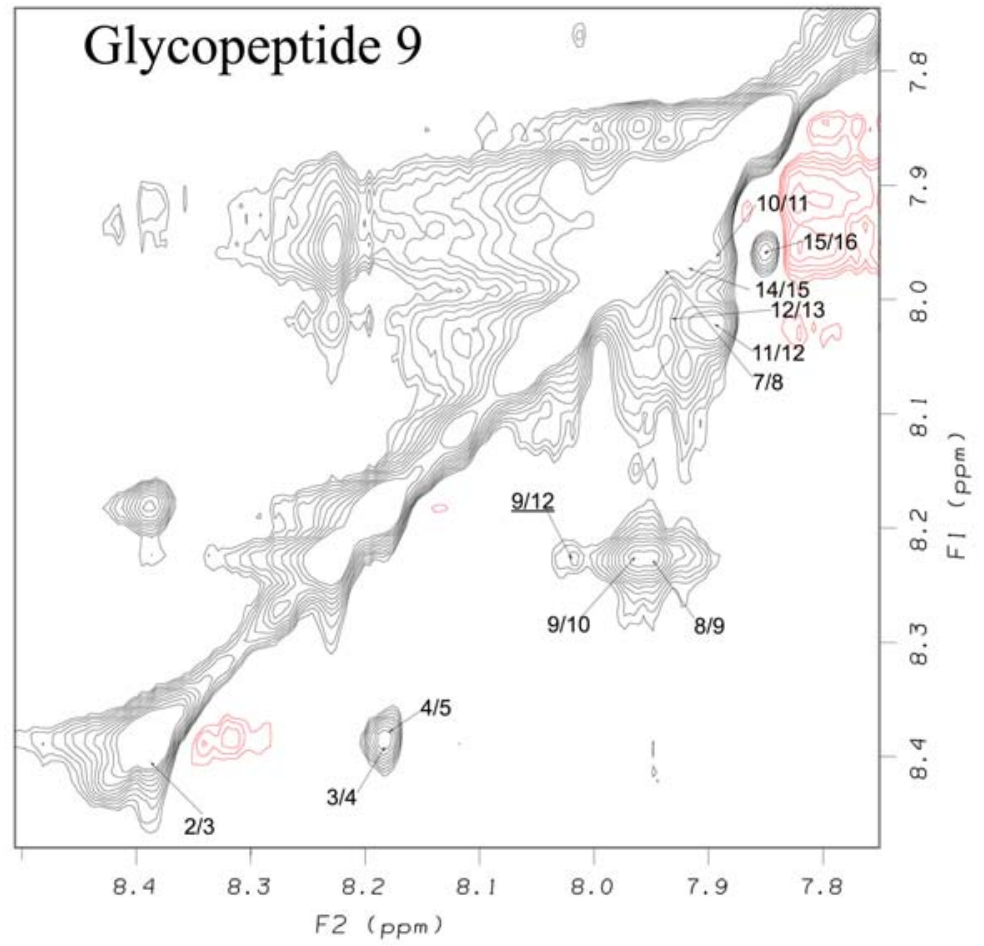

Figure S16. Amide $(\mathrm{NH}-\mathrm{NH})$ region of NOESY (mixing time $=300 \mathrm{~ms}$ ) spectra in zwitterionic bicelle at $\mathrm{pH}=4.5$ and $38^{\circ} \mathrm{C}$. 


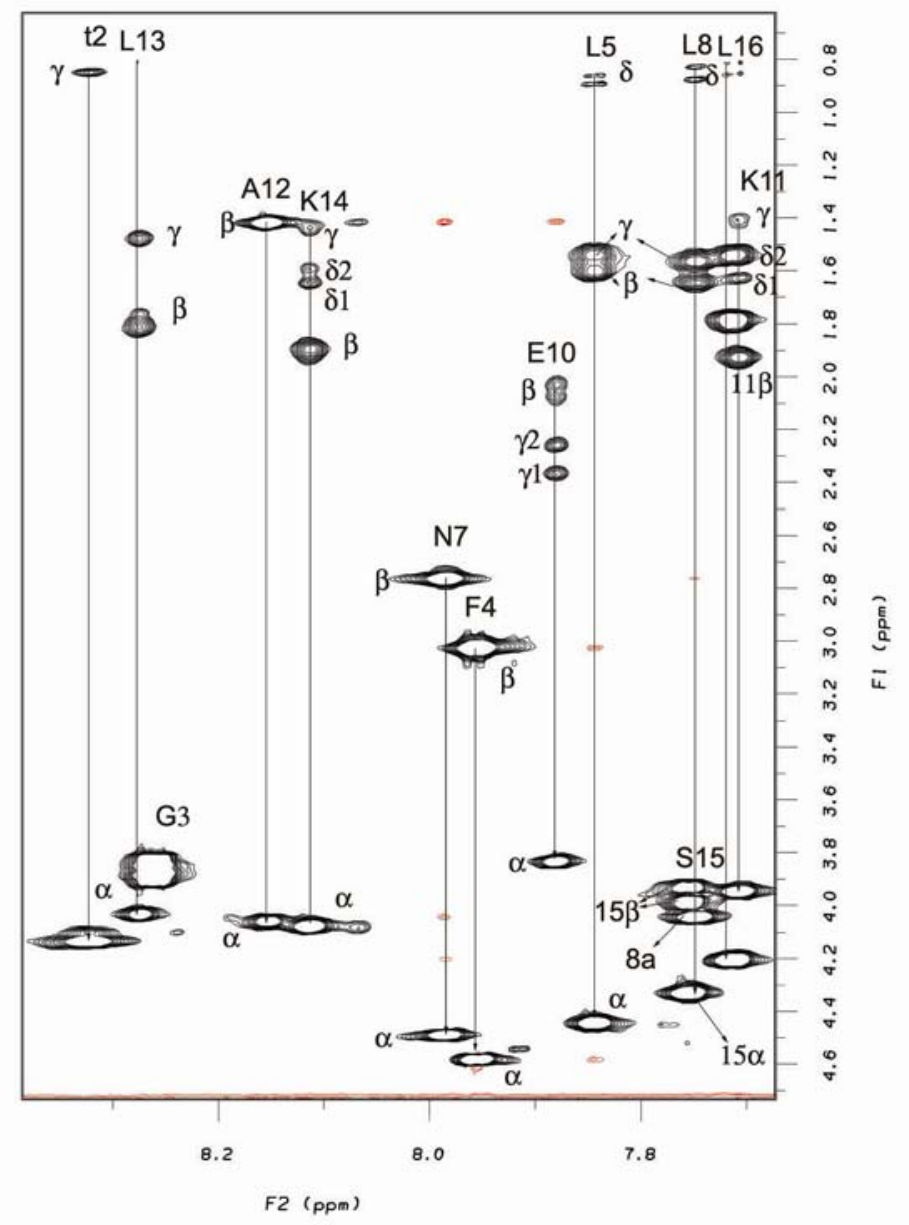

Figure S17. A portion of TOCSY (mixing time $=100 \mathrm{~ms}$ ) spectra showing all the spin system of peptide $9 \mathbf{u}$ in TFE:water (3:7) at $\mathrm{pH}=4.5$ and at $15^{\circ} \mathrm{C}$. 


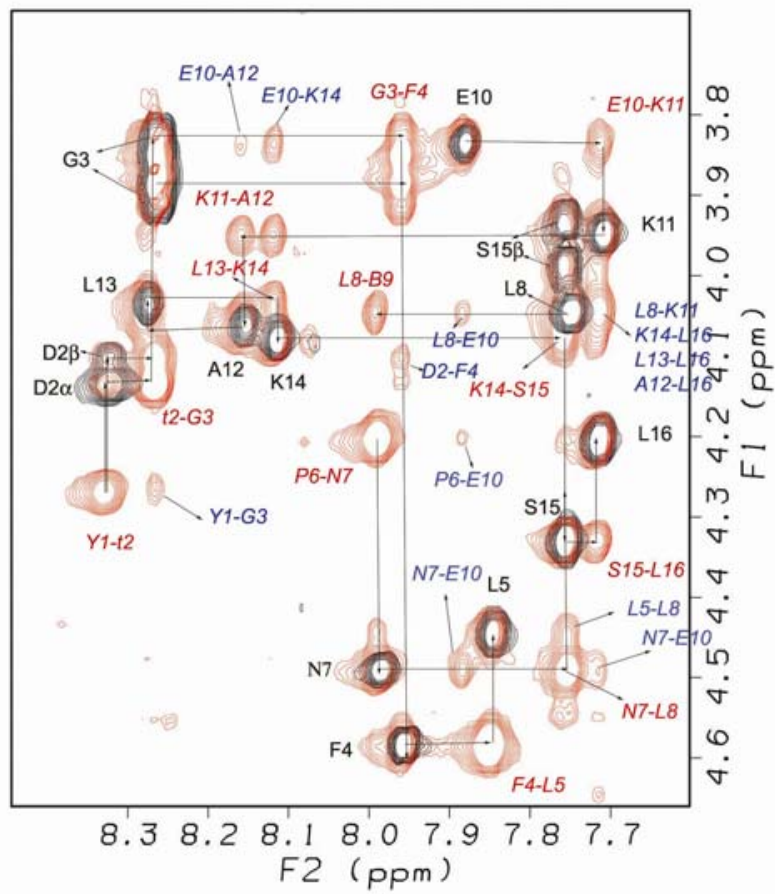

Figure S18. Finger print $(\alpha \mathrm{H}-\mathrm{NH})$ region of TOCSY (in black colour, mixing time $=$ $100 \mathrm{~ms}$ ) and ROESY (in red colour, mixing time $=150 \mathrm{~ms}$ ) of peptide $9 \mathbf{u}$ in TFE:water (3:7) at $\mathrm{pH}=4.5$ and at $15^{\circ} \mathrm{C}$.

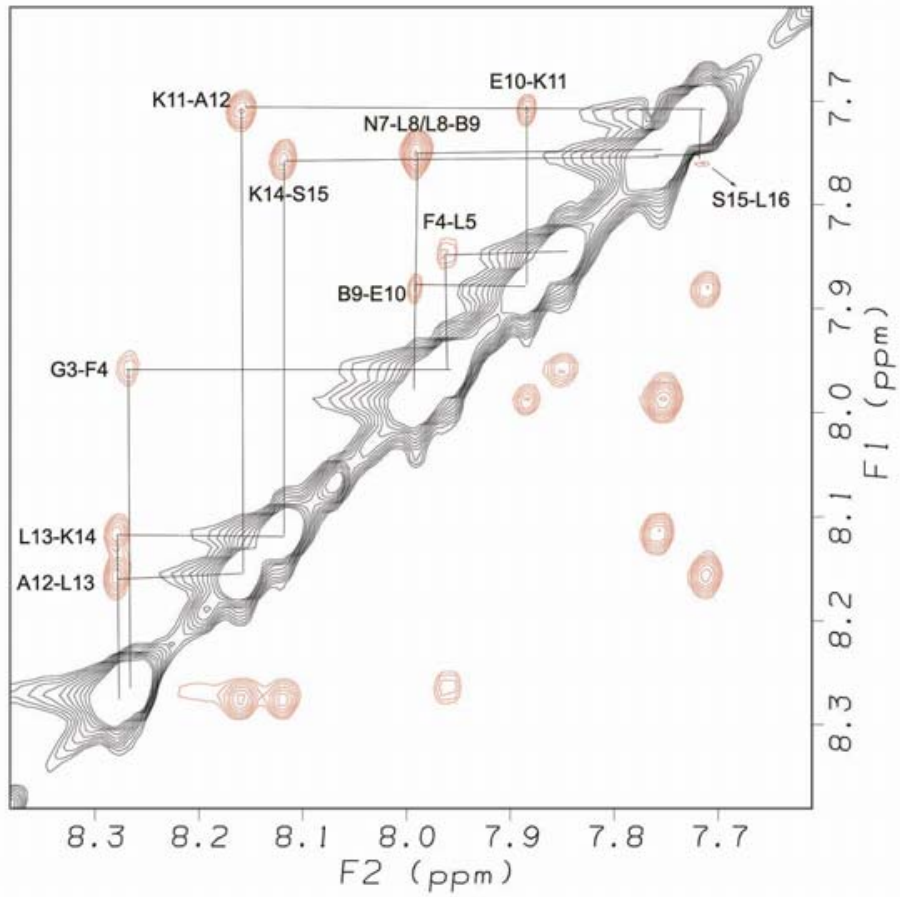

Figure S19. Amide (NH-NH) region of ROESY (mixing time $=150 \mathrm{~ms}$ ) spectra of peptide $9 \mathbf{u}$ in TFE:water $(3: 7)$ at $\mathrm{pH}=4.5$ and at $15^{\circ} \mathrm{C}$. 


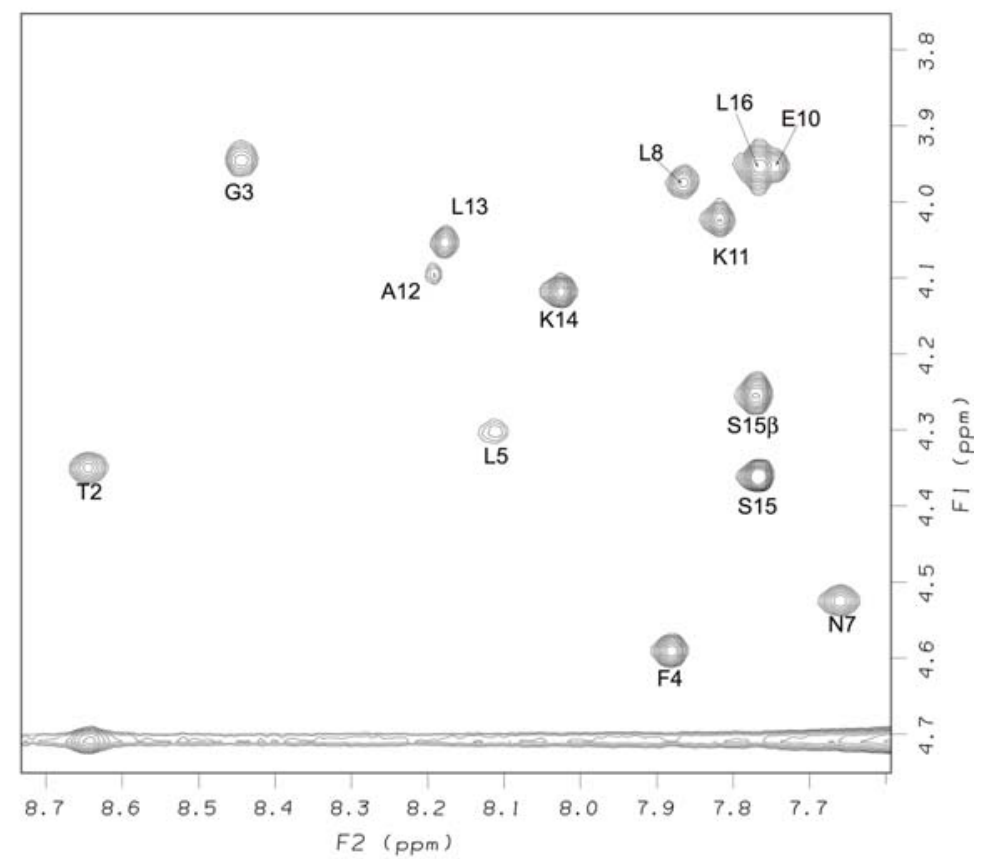

a)

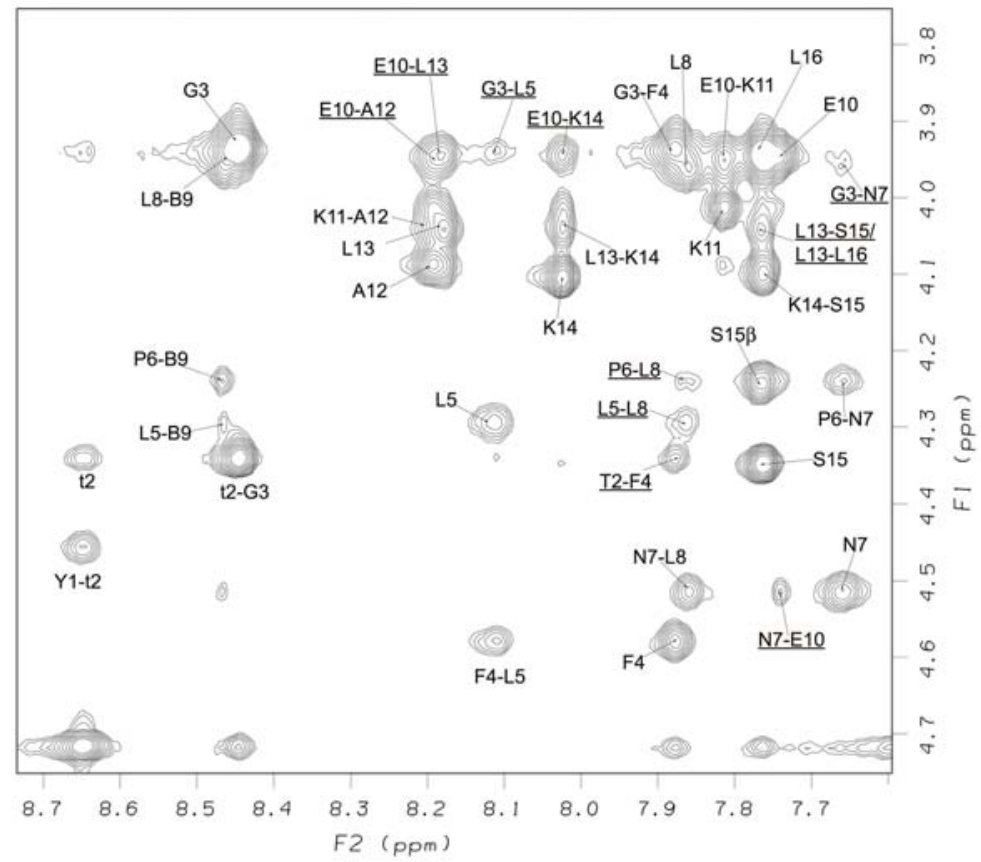

b)

Figure S20: Finger print $(\alpha \mathrm{H}-\mathrm{NH})$ region of a) TOCSY (mixing time $=100 \mathrm{~ms}$ ) and $\mathrm{b}$ ) NOESY (mixing time $=300 \mathrm{~ms}$ ) of peptide $9 \mathbf{u}$ in SDS micelles at $\mathrm{pH}=4.5$ and at $25^{\circ} \mathrm{C}$. 


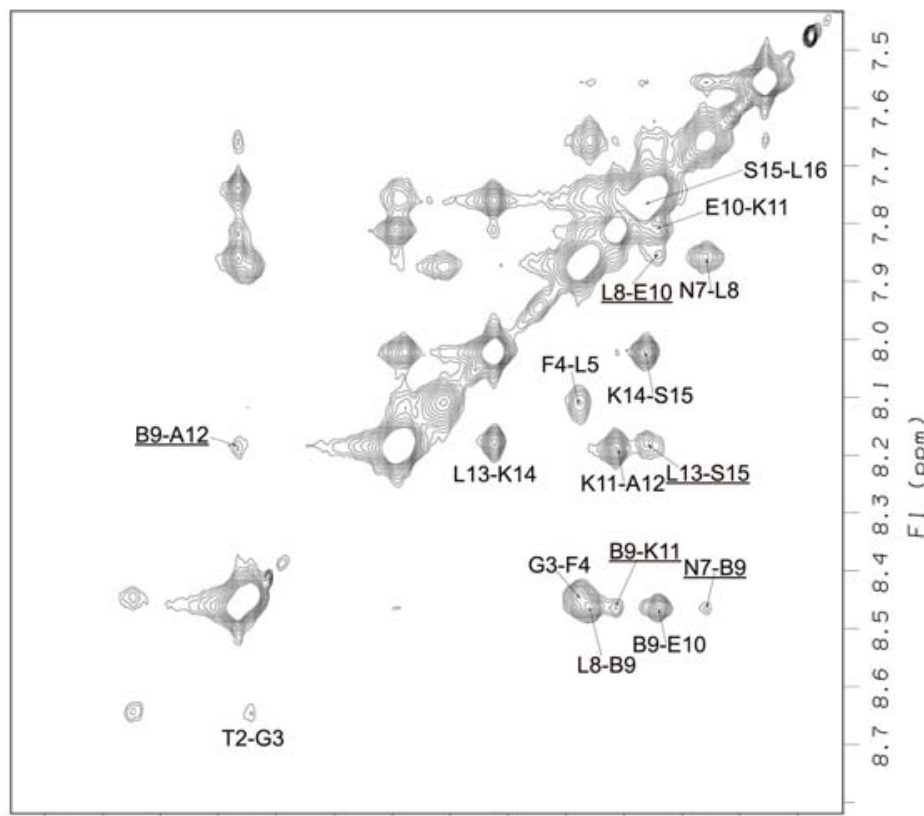

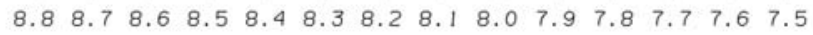

F2 (ppm)

Figure S21. Amide $(\mathrm{NH}-\mathrm{NH})$ region of NOESY (mixing time $=300 \mathrm{~ms}$ ) spectra of peptide $9 \mathbf{u}$ in SDS micelles at $\mathrm{pH}=4.5$ and at $25^{\circ} \mathrm{C}$.

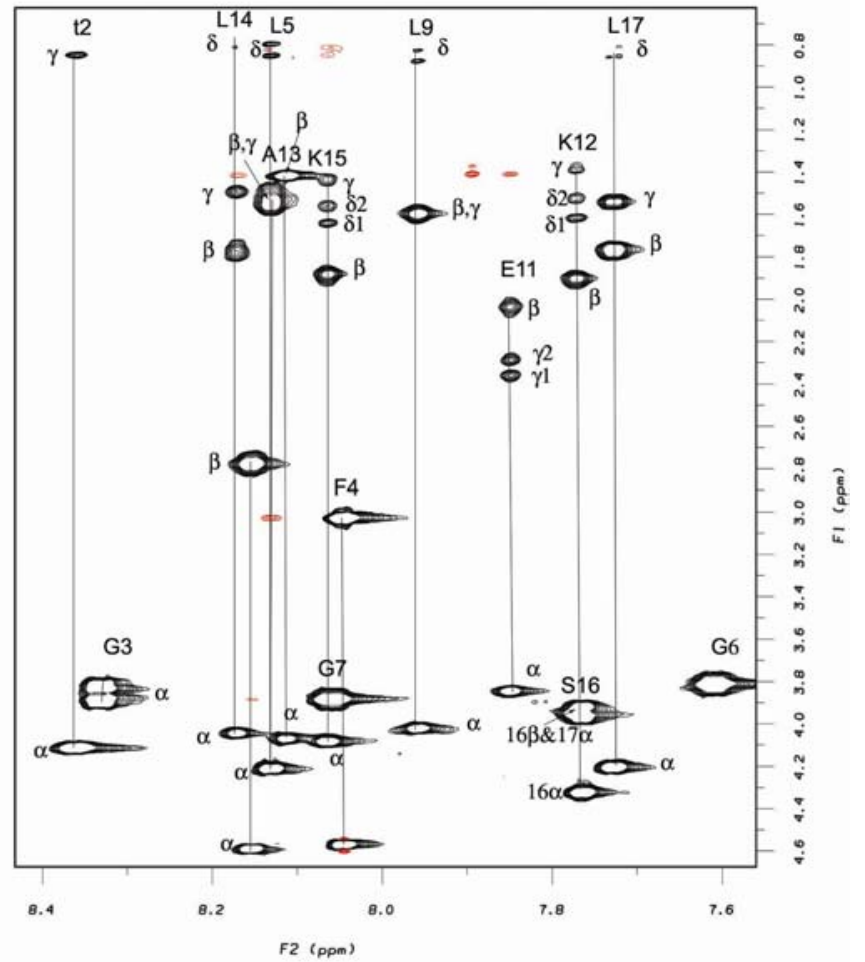

Figure S22. A portion of TOCSY (mixing time $=100 \mathrm{~ms}$ ) spectra showing all the spin system of peptide 11u in TFE:water (3:7) at $\mathrm{pH}=4.5$ and at $15^{\circ} \mathrm{C}$. 


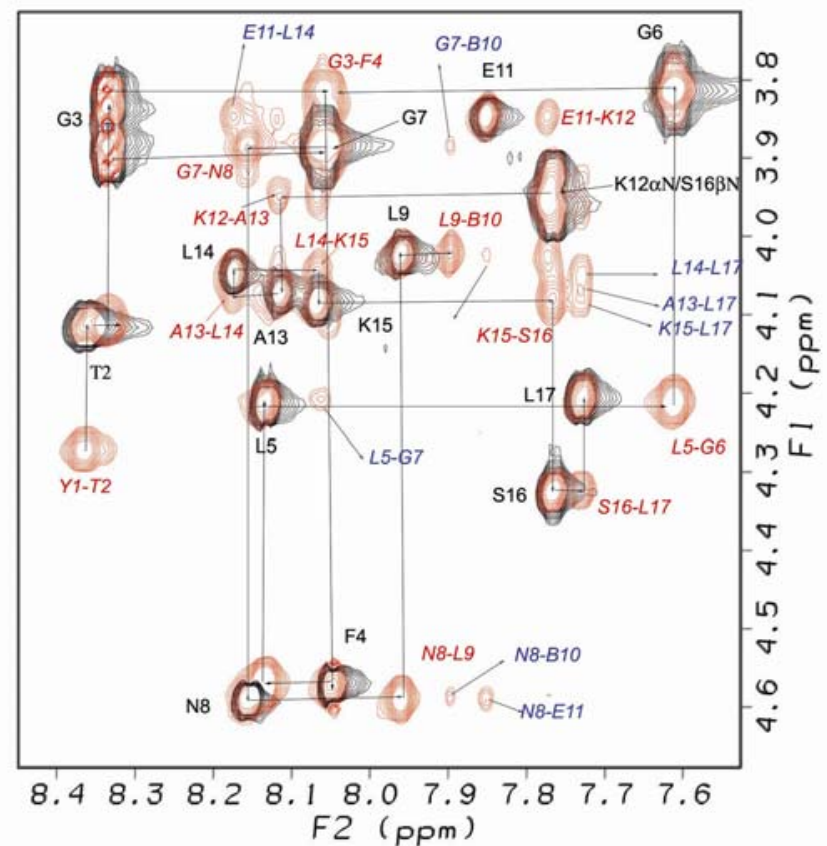

Figure S23. Finger print $(\alpha \mathrm{H}-\mathrm{NH})$ region of TOCSY (in black colour, mixing time $=$ $100 \mathrm{~ms}$ ) and ROESY (in red colour, mixing time $=150 \mathrm{~ms}$ ) of peptide $11 \mathbf{u}$ in TFE:water (3:7) at $\mathrm{pH}=4.5$ and at $15^{\circ} \mathrm{C}$.

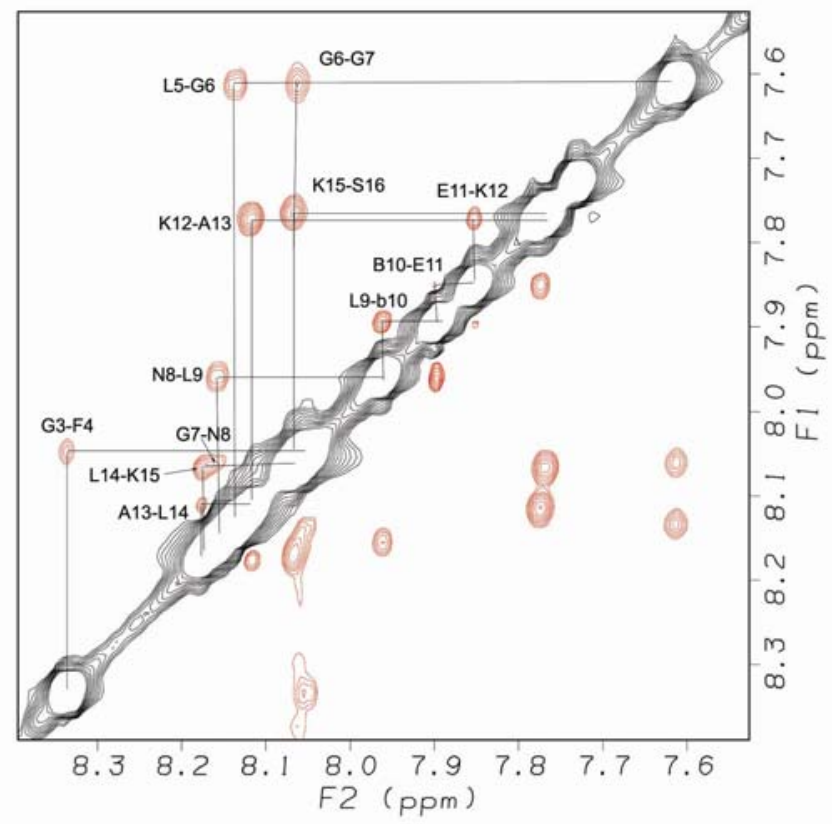

Figure S24. Amide (NH-NH) region of ROESY (mixing time $=150 \mathrm{~ms}$ ) spectra of peptide $11 \mathrm{u}$ in TFE:water $(3: 7)$ at $\mathrm{pH}=4.5$ and at $15^{\circ} \mathrm{C}$. 


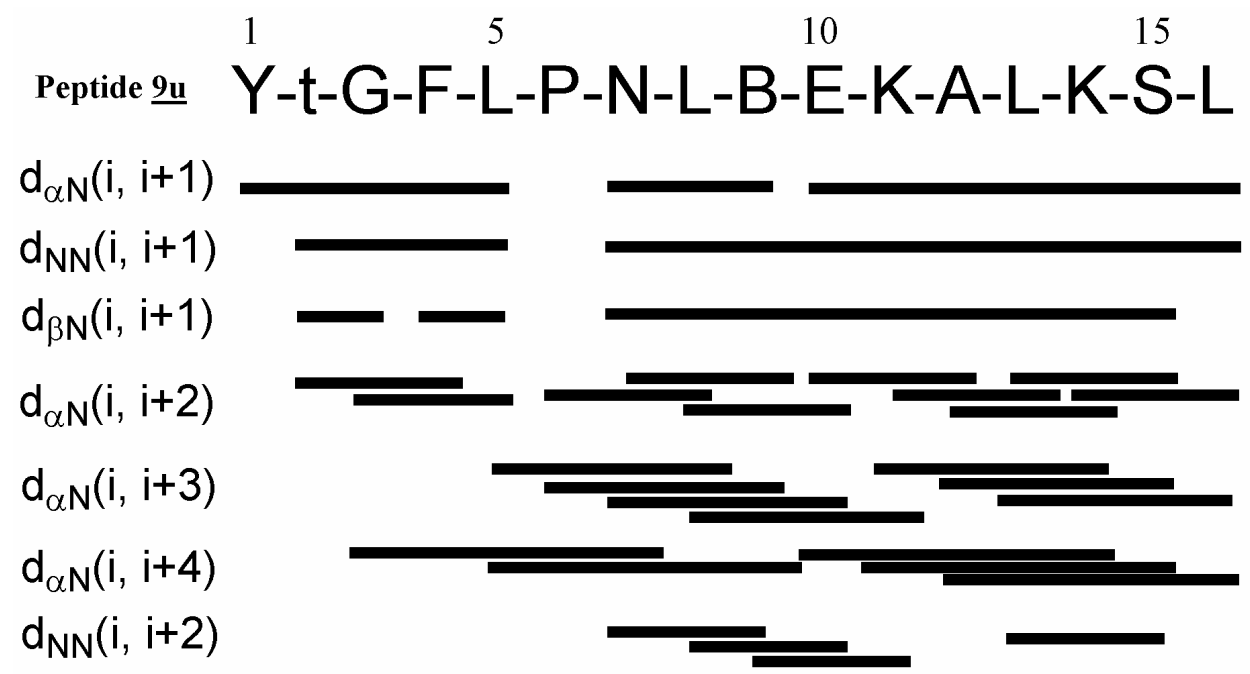

Figure S25. Summary of NOEs observed for peptide 9u in SDS micelle at $\mathrm{pH}=4.5$ and $25^{\circ} \mathrm{C}$.
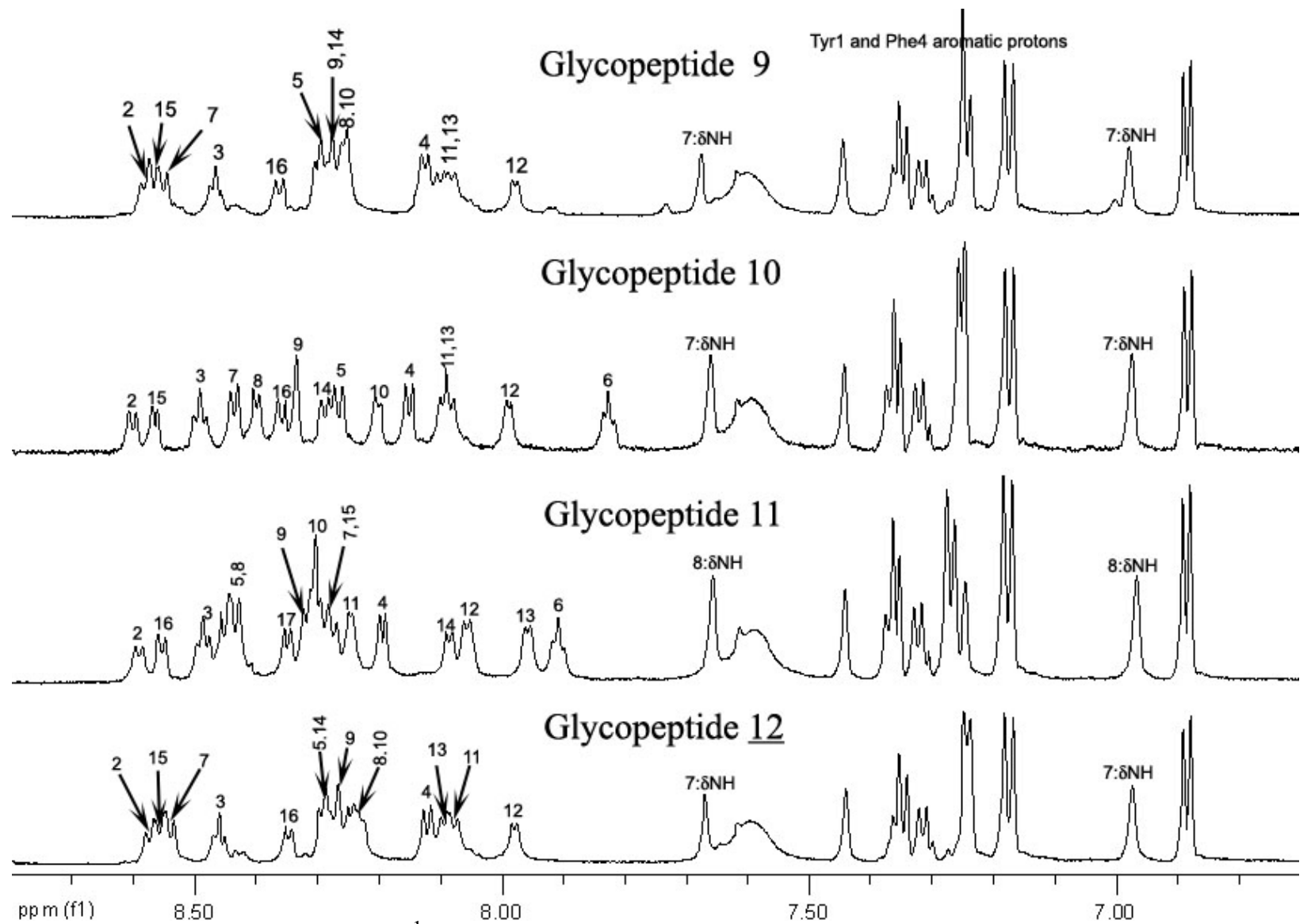

Figure S26. A portion of ${ }^{1} \mathrm{H}$ NMR spectra displaying amide and aromatic proton assignments in $\mathrm{H}_{2} \mathrm{O} / \mathrm{D}_{2} \mathrm{O}(9: 1)$ at $\mathrm{pH}=4.5$ and $20^{\circ} \mathrm{C}$. Sequence numbers from the glycopeptide are shown on the $\mathrm{NH}$ resonances. 

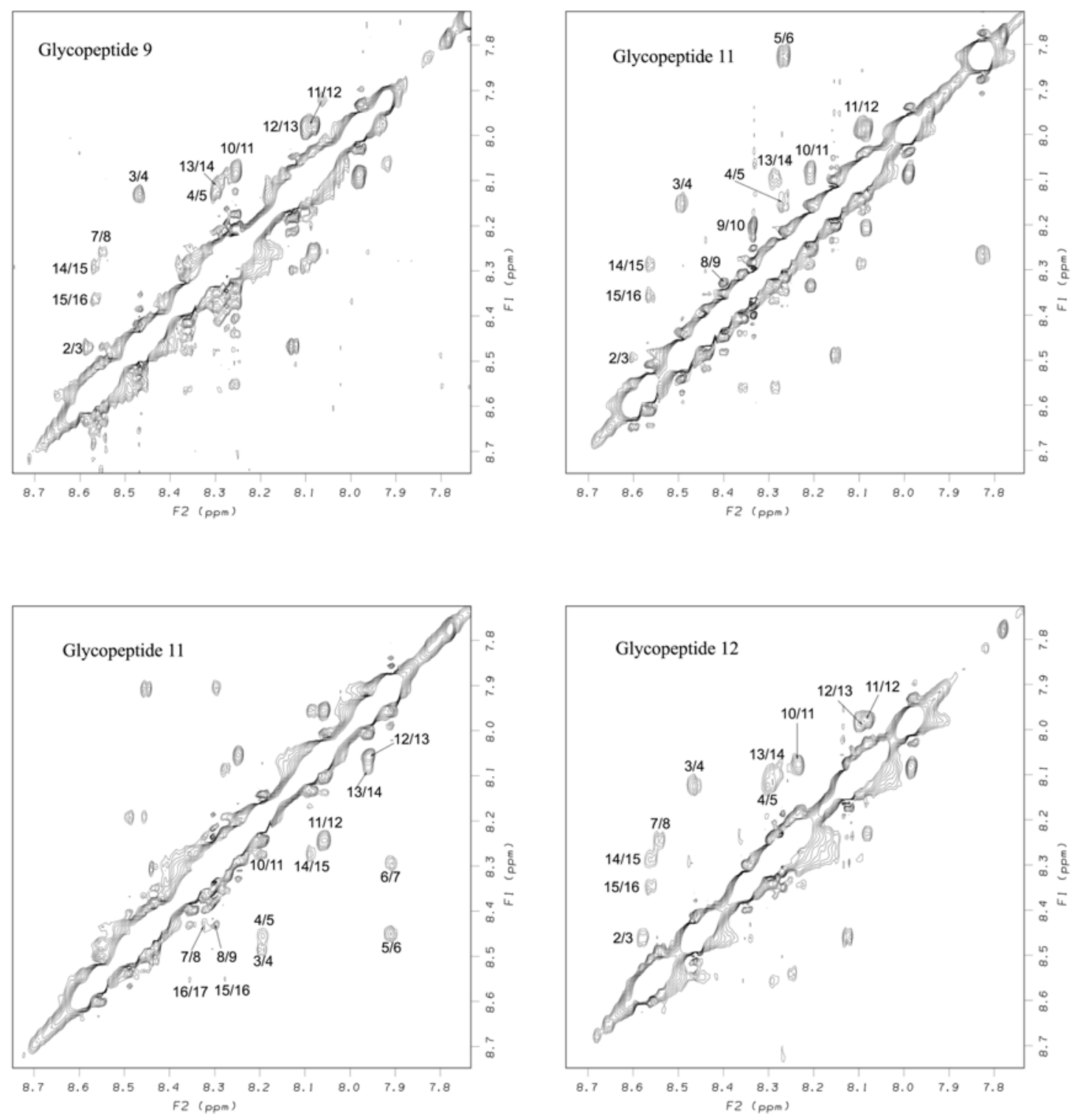

Figure S27. Amide $(\mathrm{NH}-\mathrm{NH})$ region of ROESY (mixing time $=150 \mathrm{~ms}$ ) spectra displaying $\mathrm{d}_{\mathrm{NN}}(\mathrm{i}, \mathrm{i}+1)$ ROE peaks in $\mathrm{H}_{2} \mathrm{O}: \mathrm{D}_{2} \mathrm{O}(9: 1)$ at $\mathrm{pH}=4.5$ at $20^{\circ} \mathrm{C}$. Observation of sequential $\mathrm{d}_{\mathrm{NN}}(\mathrm{i}, \mathrm{i}+1)$ NOEs indicates helical conformations. 

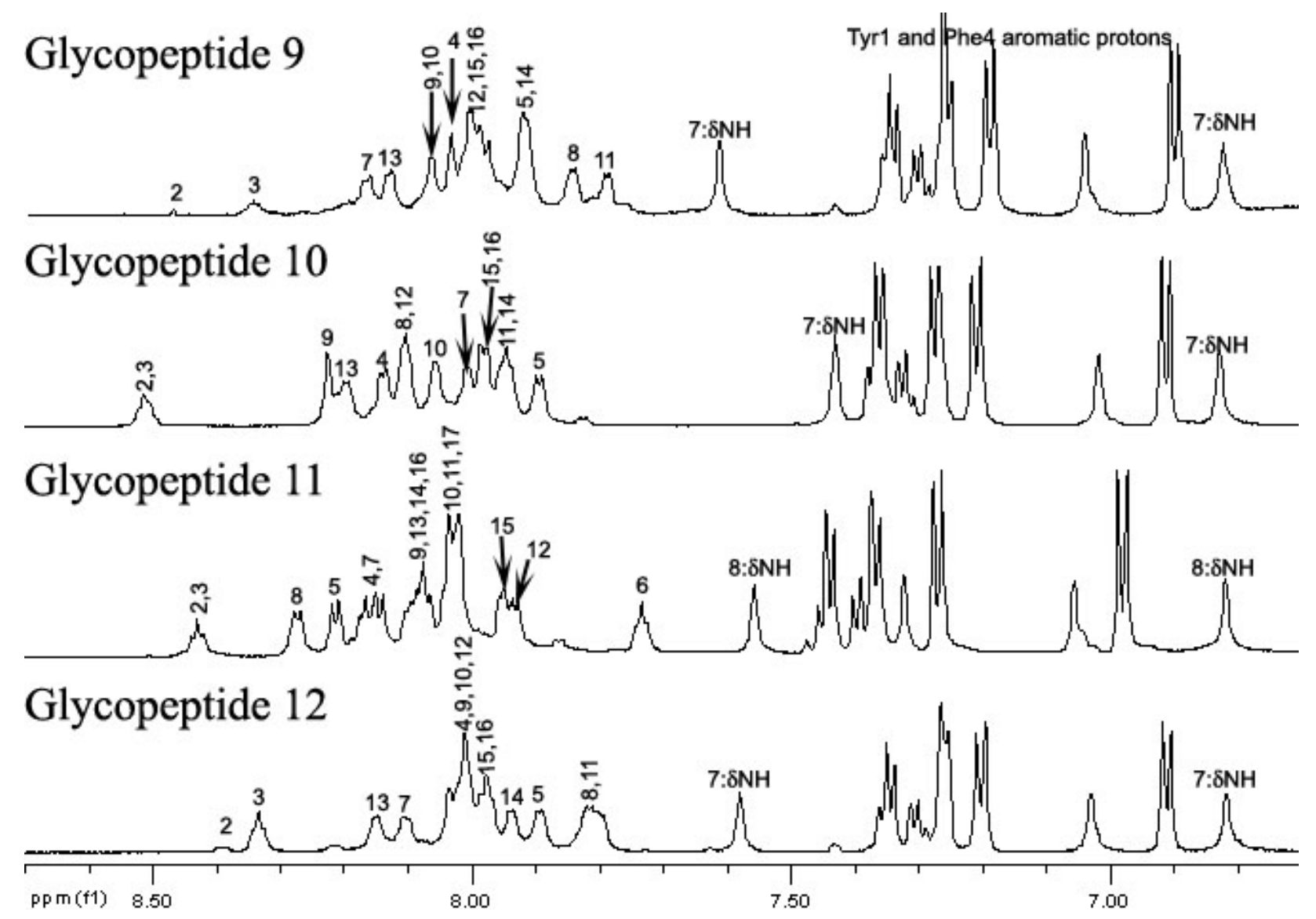

Figure S28. A portion of ${ }^{1} \mathrm{H}$ NMR spectra displaying amide and aromatic proton assignments in $\mathrm{TFE} / \mathrm{H}_{2} \mathrm{O}(3: 7)$ and $\mathrm{pH}=4.5$ at $15^{\circ} \mathrm{C}$. Sequence numbers from the glycopeptide are shown at the $\mathrm{NH}$ resonances. 

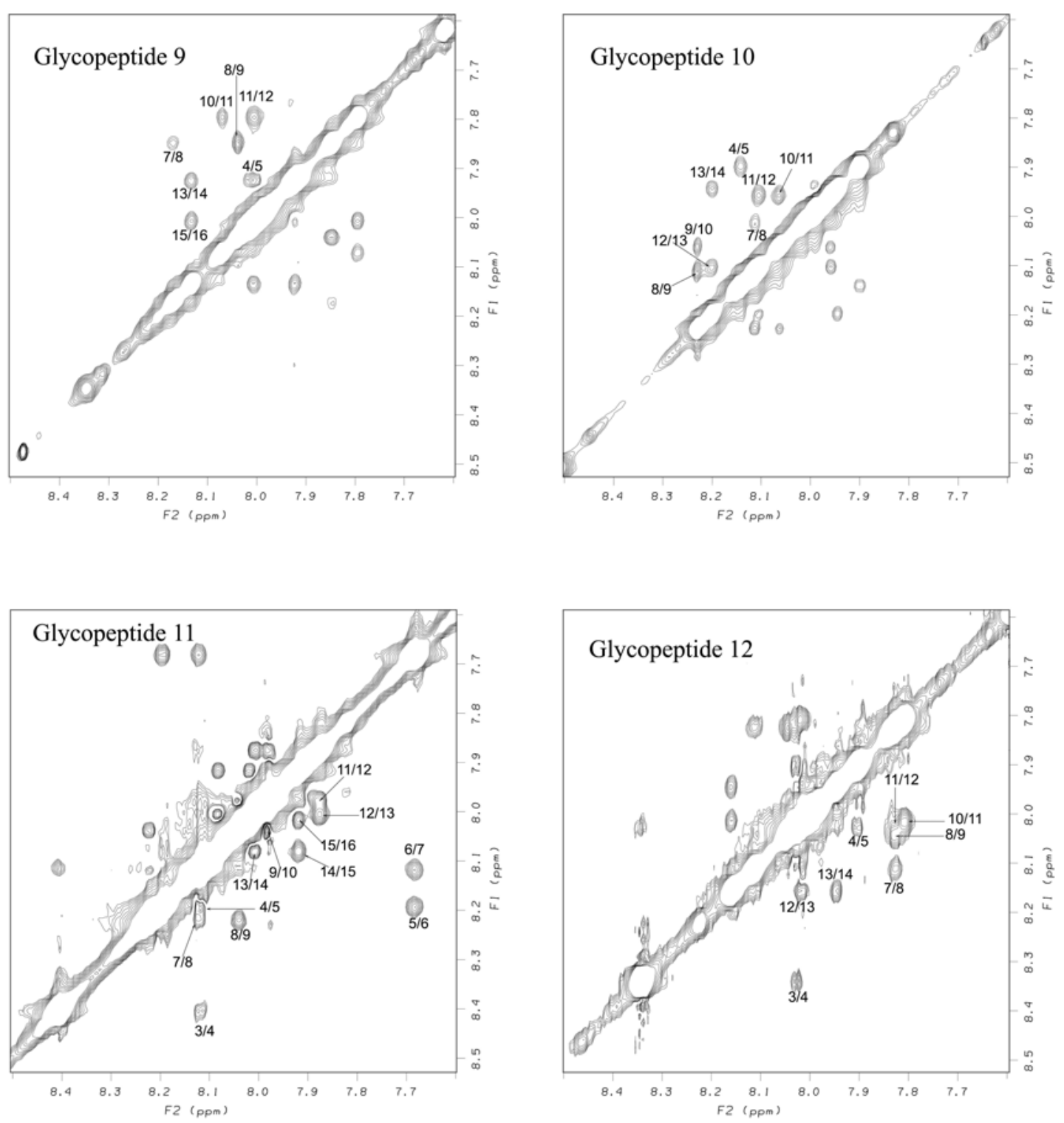

Figure S29. Amide $(\mathrm{NH}-\mathrm{NH})$ region of ROESY (mixing time $=150 \mathrm{msec}$ ) displaying $\mathrm{d}_{\mathrm{NN}}(\mathrm{i}, \mathrm{i}+1)$ NOEs spectra in $\mathrm{TFE} / \mathrm{H}_{2} \mathrm{O}(3: 7)$ and $\mathrm{pH}=4.5$ at $15^{\circ} \mathrm{C}$. Observation of sequential $d_{N N}(i, i+1)$ NOEs indicates helical conformation. Some of the potential $d_{N N}(i$, i+1) NOEs are too close to diagonal peak to observe. 

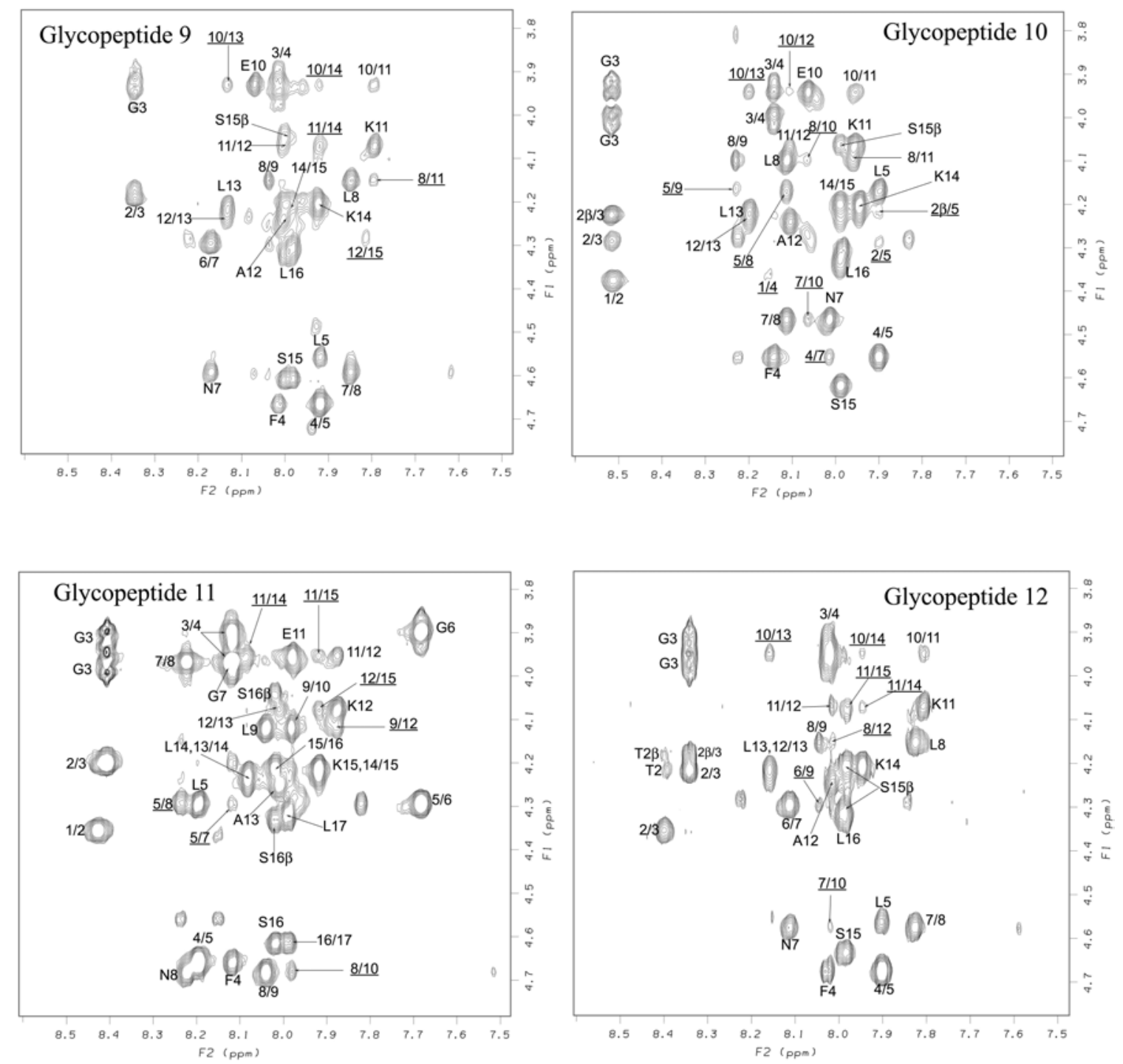

Figure S30. Fingerprint region $(\alpha \mathrm{H}-\mathrm{NH})$ of ROESY (mixing time $=150 \mathrm{~ms}$ ) spectra in TFE:water (3:7) and $\mathrm{pH}=4.5$ at $15^{\circ} \mathrm{C}$. The $\mathrm{d}_{\alpha \mathrm{N}}(\mathrm{i}, \mathrm{i}+2), \mathrm{d}_{\alpha \mathrm{N}}(\mathrm{i}, \mathrm{i}+3)$ and $\mathrm{d}_{\alpha \mathrm{N}}(\mathrm{i}, \mathrm{i}+4)$ ROEs are underlined. 
Glycopeptide 9

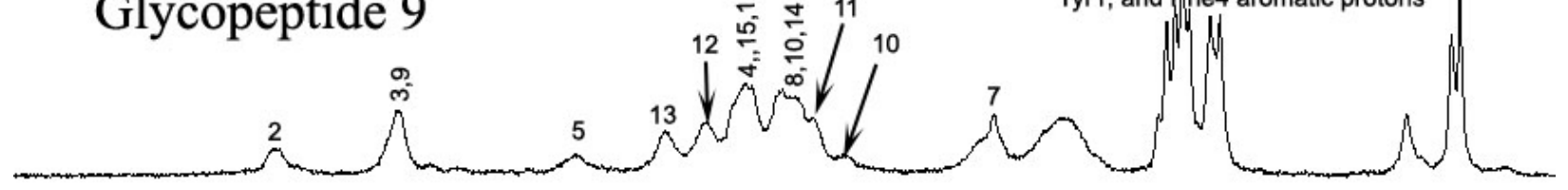

Glycoeptide 10

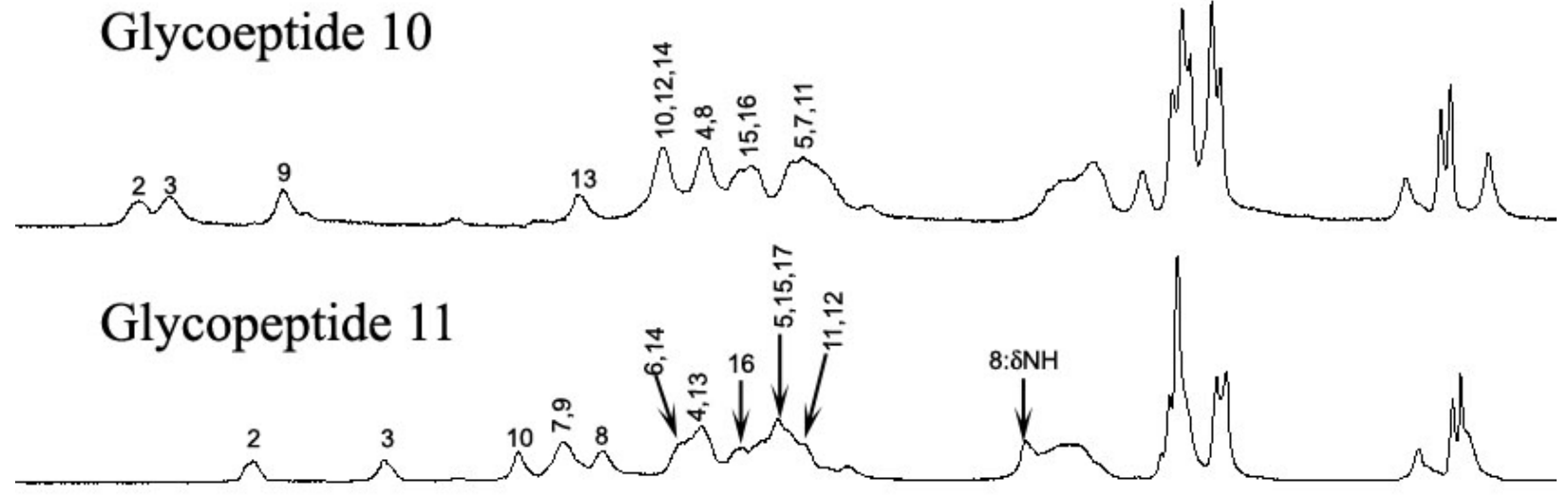

Glycopeptide 12

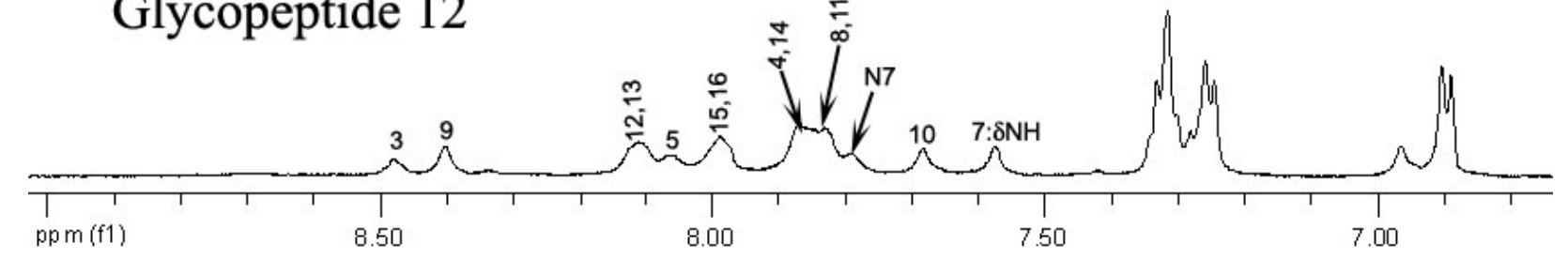

Figure S31. A portion of ${ }^{1} \mathrm{H}$ NMR spectra displaying amide and aromatic proton assignments in SDS micelles at $\mathrm{pH}=4.5$ at $25^{\circ} \mathrm{C}$. Sequence numbers from the glycopeptide are shown on the $\mathrm{NH}$ resonances. The glycopeptide-to-SDS micelle ratio was 1:100. 

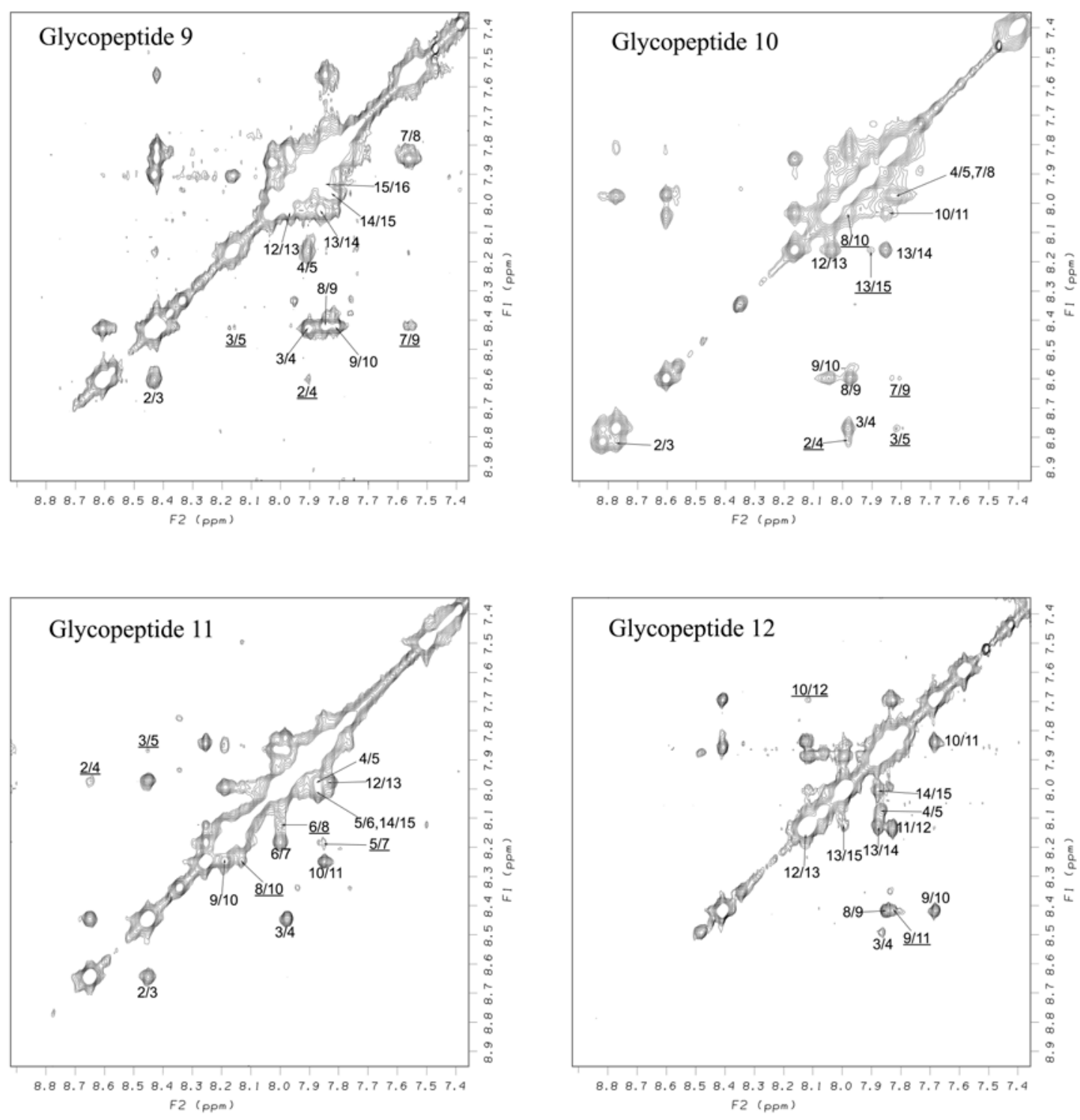

Figure S32. Amide region $(\mathrm{NH}-\mathrm{NH})$ of NOESY (mixing time $=300 \mathrm{msec}$ ) spectra in SDS micelles at $\mathrm{pH}=4.5$ and $25^{\circ} \mathrm{C}$. Observation of sequential $\mathrm{d}_{\mathrm{NN}}(\mathrm{i}, \mathrm{i}+1)$ NOEs indicates helical conformation. The helical signature $\mathrm{d}_{\mathrm{NN}}(\mathrm{i}, \mathrm{i}+2)$ NOEs are underlined. 

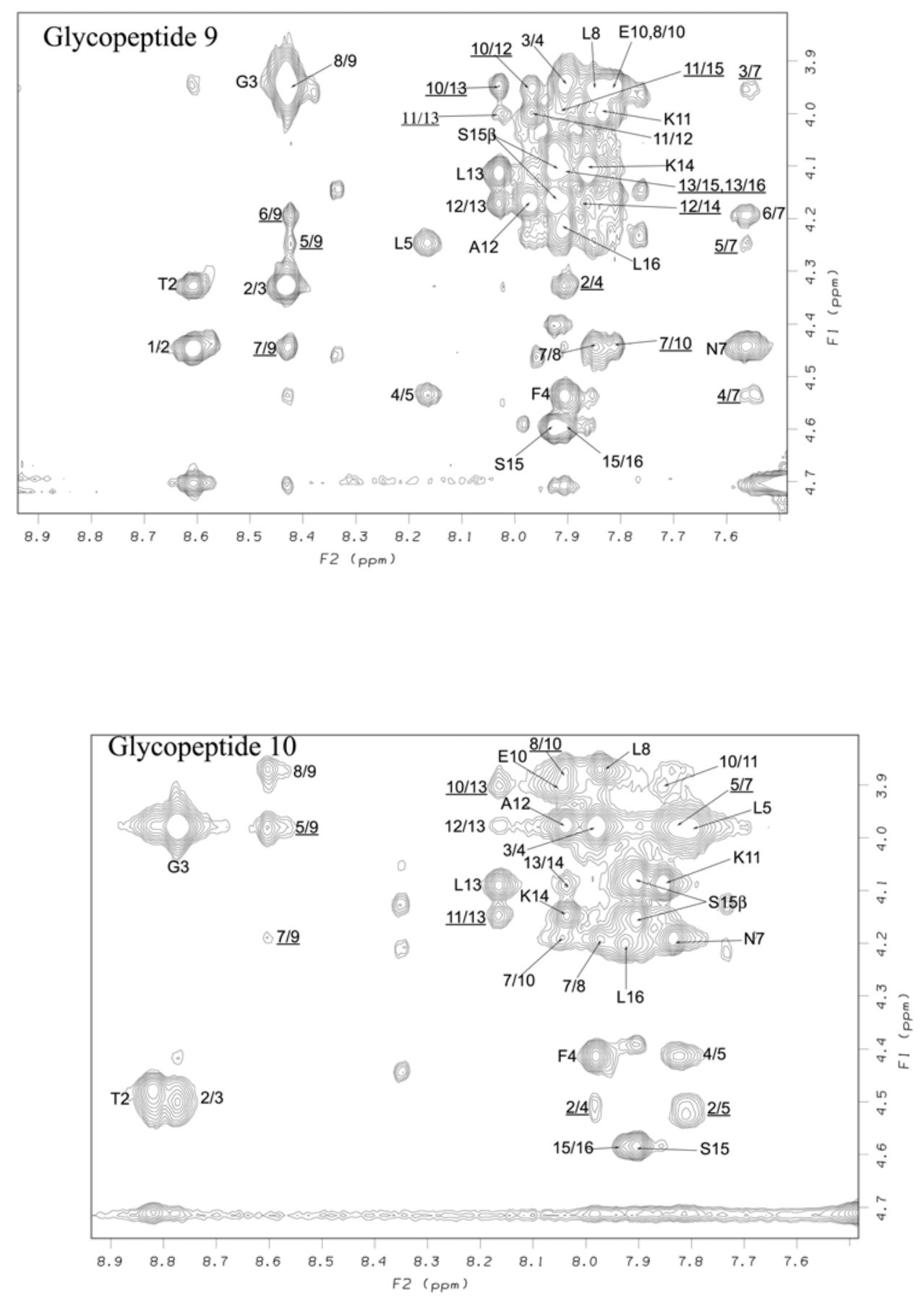

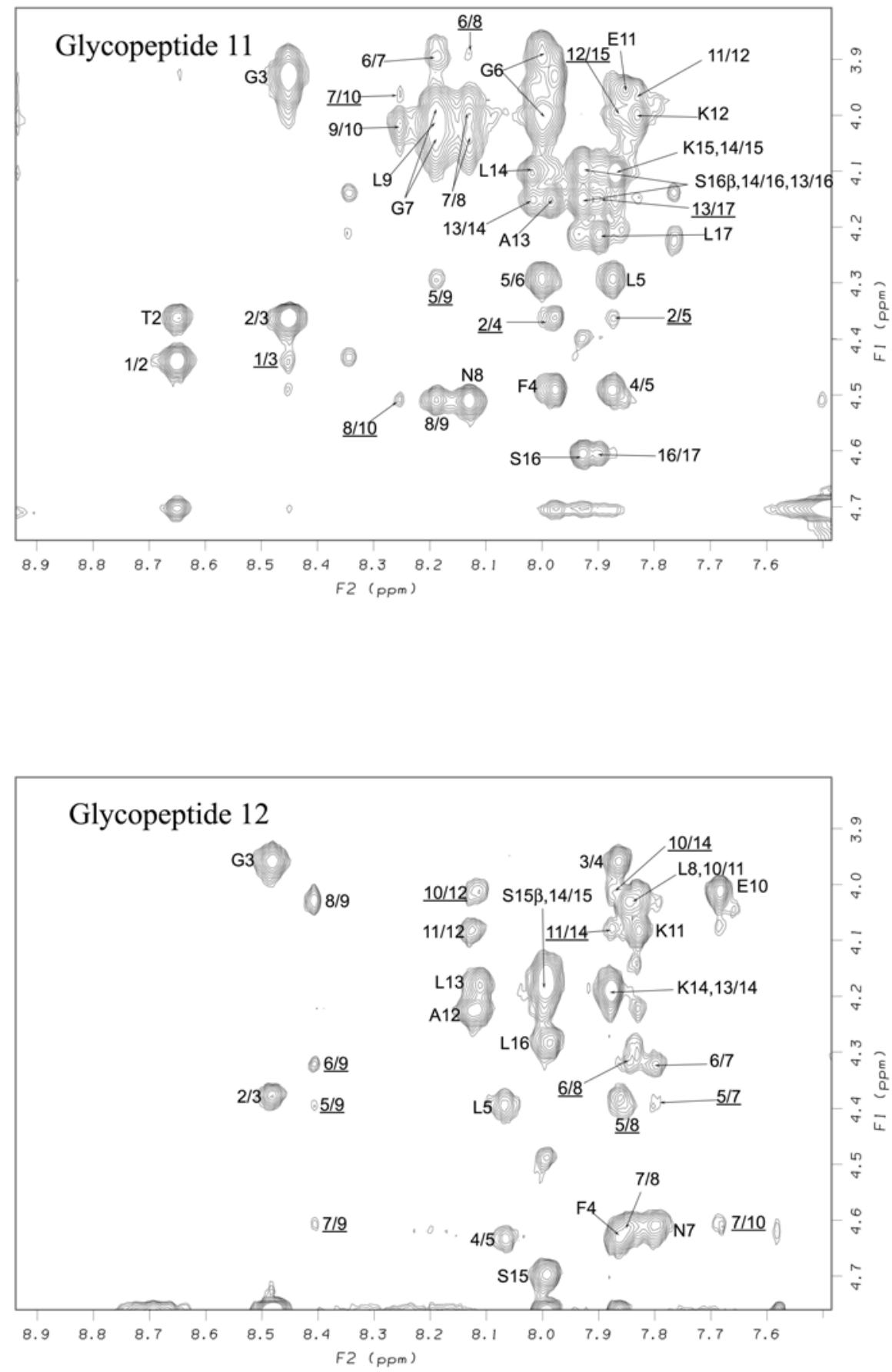

Figure S33. Finger print region of $(\alpha \mathrm{H}-\mathrm{NH})$ of NOESY (mixing time $=300 \mathrm{msec}$ ) spectra in SDS micelles at $\mathrm{pH}=4.5$ and $25^{\circ} \mathrm{C}$. The medium and long range NOEs are underlined. 
Glycopeptide 9
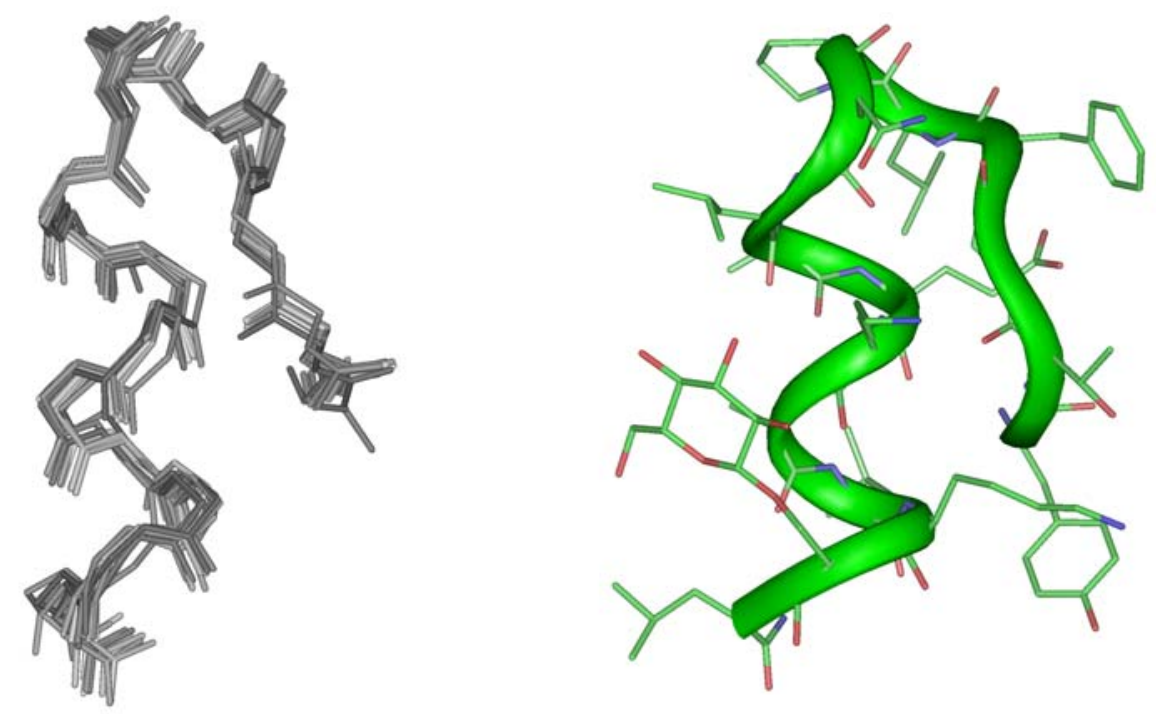

Glycopeptide 10
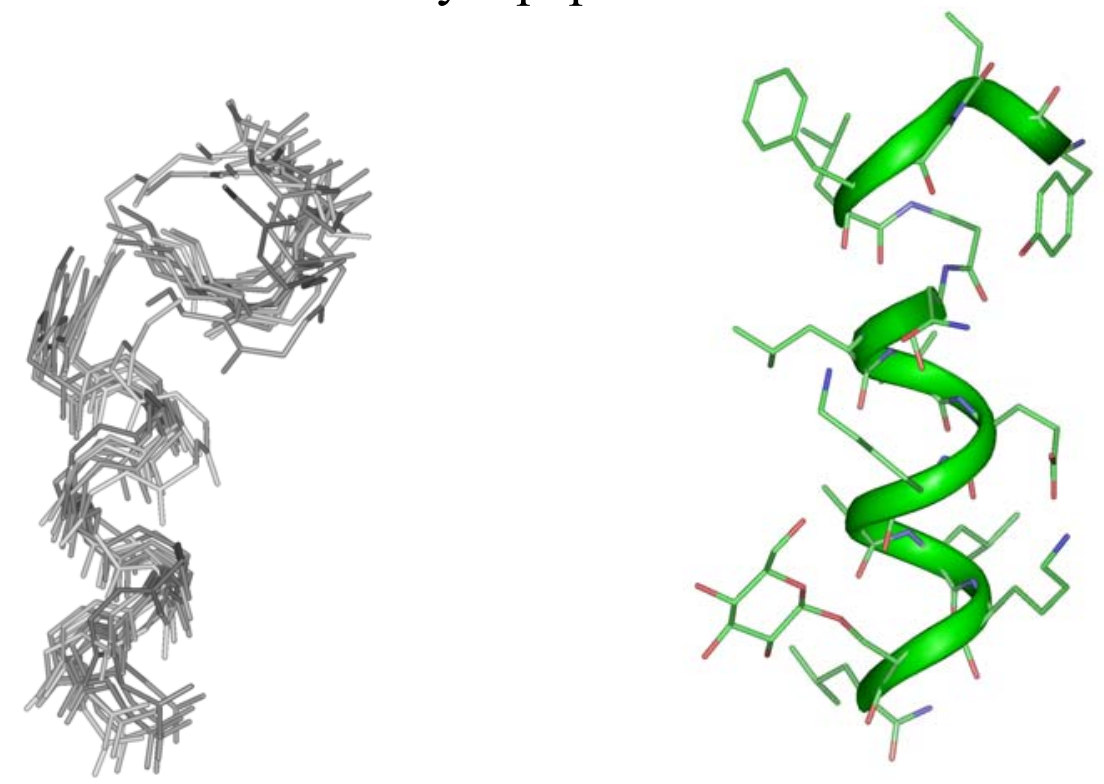

Glycopeptide 11 


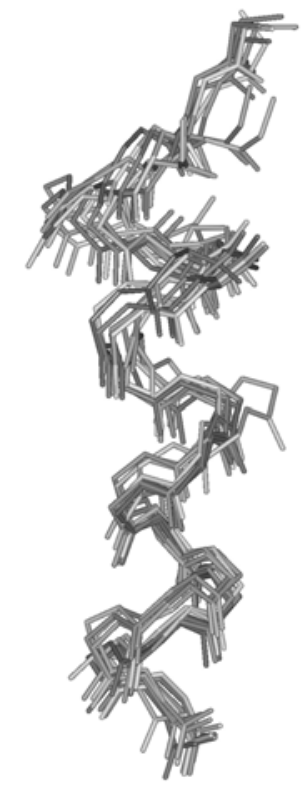

\section{Glycopeptide 12}
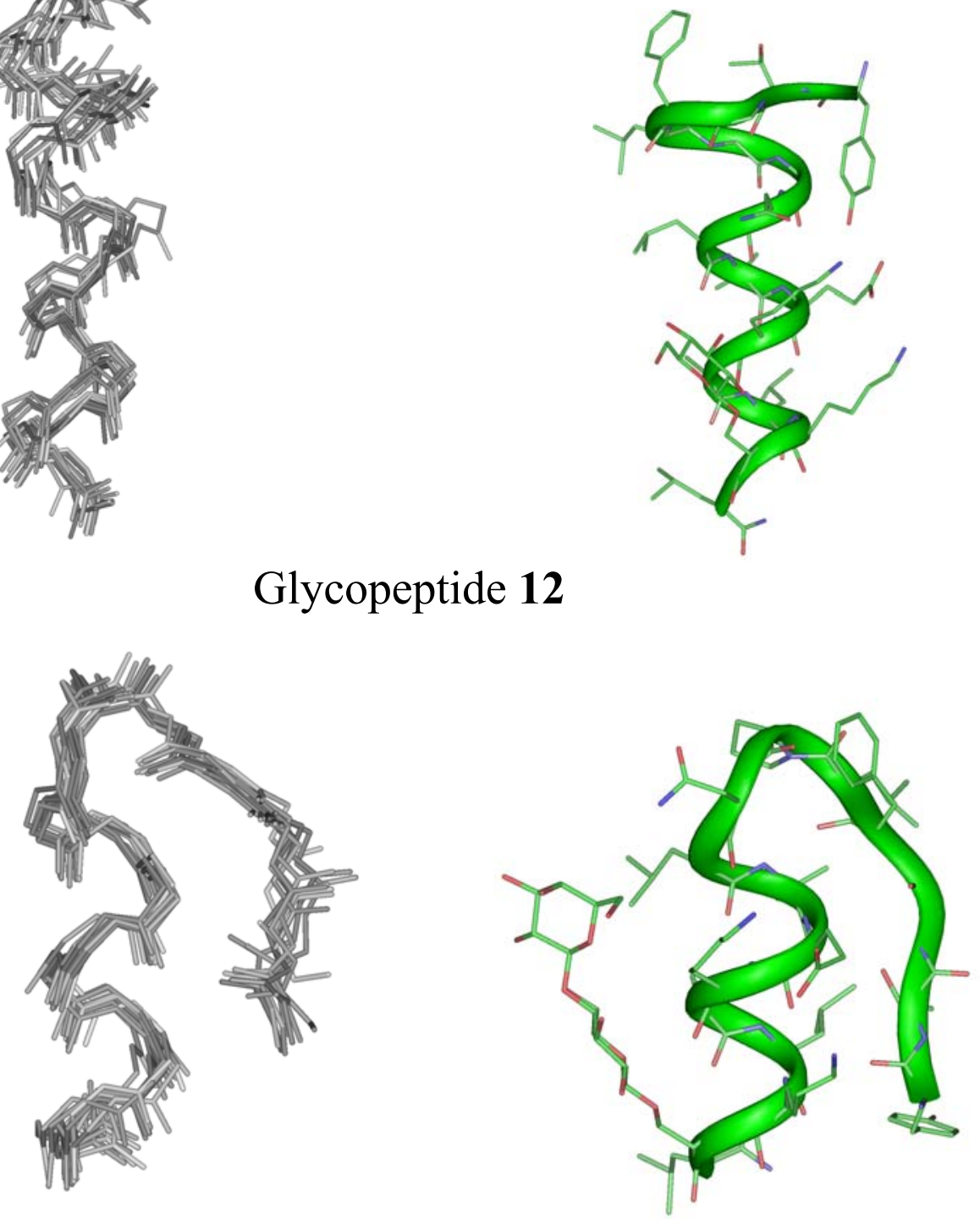

a)

b)

Figure S34. a) An overlay of the ten lowest energy structures from the simulated annealing molecular dynamics calculations using NOE data from SDS micelles media. The glycoside moieties have been omitted for clarity. b) Ribbon representations of the lowest energy structure for $\mathbf{9 - 1 2}$. 